\title{
Random Particle Methods Applied to Broadband Fan Interaction Noise
}

\author{
M. Dieste, G. Gabard \\ Institute of Sound and Vibration Research, University of Southampton, SO17 1BJ, U.K. \\ gabard@soton.ac.uk
}

\begin{abstract}
Predicting broadband fan noise is key to reduce noise emissions from aircraft and wind turbines. Complete CFD simulations of broadband fan noise generation remain too expensive to be used routinely for engineering design. A more efficient approach consists in synthesizing a turbulent velocity field that captures the main features of the exact solution. This synthetic turbulence is then used in a noise source model. This paper concentrates on predicting broadband fan noise interaction (also called leading edge noise) and demonstrates that a Random Particle Mesh method (RPM) is well suited for simulating this source mechanism. The linearized Euler equations are used to describe sound generation and propagation. In this work, the definition of the filter kernel is generalized to include non-Gaussian filters that can directly follow more realistic energy spectra such as the ones developed by Liepmann and von Kármán. The velocity correlation and energy spectrum of the turbulence are found to be well captured by the RPM. The acoustic predictions are successfully validated against Amiet's analytical solution for a flat plate in a turbulent stream. A standard Langevin equation is used to model temporal decorrelation, but the presence of numerical issues leads to the introduction and validation of a second-order Langevin model.
\end{abstract}

Keywords: broadband fan noise, stochastic methods, random particle method, aero-acoustics

\section{Introduction}

Fan noise has become a major component of noise emission from modern aircraft, mainly due to an increase in bypass ratio and the associated reduction in jet noise. Tonal fan noise can be efficiently reduced by tuning the liner properties to target the blade passing frequency and its harmonics. Broadband fan noise remains however more difficult to predict and to reduce because of its random nature and wide frequency content associated with the turbulent fluctuations.

Direct Numerical Simulation (DNS) or Large Eddy Simulation (LES) are used for predicting broadband fan noise. But due to the wide range of length and time scales present in turbulent flows, this approach remains extremely expensive and has not been fully validated, and as such it is not envisioned to be used in the near future as a design and optimization tool for noise prediction. To reduce computational costs, an alternative is to use a full unsteady CFD calculation only in a limited source region and to combine the results with an aero-acoustics model for the generation and propagation of noise. It is possible to go one step further and only use a steady RANS (Reynolds Averaged Navier-Stokes) calculation for the source region, and then to synthesize an unsteady turbulent field with the same statistical properties as that predicted by the RANS simulation. The rationale for such an approach is that for engineering purposes one does not need to capture the full details of the unsteady turbulent field and only some statistical properties are required to perform sufficiently accurate predictions. To achieve this, one can use stochastic methods to synthesize random turbulent fields that are not exact solutions of the fluid dynamics but that capture key features of the sound sources, such as the energy spectrum, length and time scales, etc. The resulting synthetic field can then be combined with acoustic propagation models.

Stochastic methods to generate turbulent flows have originally been used to study scalar dissipation and to generate inflow turbulence for DNS and LES. Early attempts relied on expressing the turbulent velocity field as a finite sum of Fourier modes where parameters such as amplitudes, wavenumbers and phases are chosen randomly following given distributions. This approach was first introduced by Kraichnan [1] in 1970 and various improvements of this 
method have since been presented with the SNGR methods [2, 3, 4]. Fourier-mode models can achieve good levels of accuracy, but they are computationally demanding and are not well suited to represent inhomogeneous turbulence [5].

In the context of fan broadband noise, Fourier-mode methods have been applied in several ways. Casper and Farassat [6] developed a formulation for broadband fan noise predictions where the turbulent wall pressure along the airfoil is modeled by Fourier modes whose parameters are generated stochastically. A time-domain formulation of the Ffowcs Williams - Hawkings equation is used to predict the acoustic far field.

Atassi et al. [7] studied the effects of three-dimensional turbulence around a cascade of flat plates by introducing stochastic sources in the linearized Euler equations. The incident turbulent flow consists in a uniform base flow and an isotropic, locally homogeneous fluctuating velocity field. The model has also been extended to include nonuniform base flows [8] and different energy spectra [9]. The fluctuating components of the inflow velocity field are described as a sum of Fourier modes and the propagation model is solved in the frequency domain.

To improve computational efficiency, stochastic techniques have been developed where the turbulent field is obtained by filtering random data $[10,11,12]$. The filter is defined so that the statistical properties of the generated field match the expected properties of the turbulent flow. A significant effort to develop filter-based methods for aeroacoustic applications was led by Ewert and coworkers [13]. These methods, generally called random particle mesh (RPM) methods, have been applied to a variety of problems in aero-acoustics (see the recent review by Ewert [13]) but the case of broadband fan interaction noise has not been tackled yet.

This paper aims at assessing the use of a filter-based stochastic method to predict broadband interaction noise between a turbulent stream and an airfoil. The method used in this work is a variant of the random particle method, following the ideas developed by Careta et al. [10] and Ewert et al. [14, 15]. In the present work we will concentrate on several aspects.

Gaussian filters are commonly used in RPMs, but we consider here the use of non-Gaussian filters to generate directly different energy spectra including the Liepmann and von Kármán spectra. We will show that these new filters can achieve accurate results.

We consider both frozen and evolving turbulence. In the context of RPMs, the assumption of frozen turbulence means that the turbulent velocity is fixed when seen in a frame of reference moving with the mean flow. For evolving turbulence, stochastic Langevin equations are used to include the effect of the integral time scale of the turbulence. However, it will be shown that a standard Langevin equation using a Wiener process for the diffusion term is not suitable when coupled with a time-domain solver for the linearized Euler equations due to the lack of smoothness of the resulting synthetic velocity field. This issue has already been noticed in a different context [16], but we provide here an analysis and validation of a second-order Langevin equation that is capable of providing accurate predictions.

In this work a fully Lagrangian formulation is used to represent the vorticity and to compute the induced velocity field. This is in contrast with [17] where an auxiliary grid is used to compute the velocity field.

To demonstrate the ability of the numerical method to predict broadband fan interaction noise, the test case of a flat plate interacting with isotropic homogeneous turbulence is considered. The validation is performed using a modified version of the analytical model derived by Amiet [18].

This paper is structured as follows. In the next section the random particle method is described in detail so as to introduce the features listed above. In section 3 the method is combined with the linearized Euler equations to predict broadband fan noise. Numerical results are presented and compared against an analytical solution first for frozen turbulence in section 4 then for evolving turbulence in section 5.

\section{Random particle method}

The stochastic method presented here is a filter-based model that is able to reproduce two-dimensional, isotropic, locally homogeneous evolving turbulent flows. It builds upon the models proposed by Careta et al. [10] and Ewert et al. $[14,15]$ where the velocity field is defined in terms of a stream function that is obtained by filtering random data. The method requires as input several statistical properties of the turbulent flow such as the energy spectrum or velocity correlation, integral length and time scales. These properties can either be modeled using empirical laws, measured or predicted using RANS simulations. 


\subsection{Description of the method}

We consider a two-dimensional, incompressible turbulent flow whose velocity field $\mathbf{u}$ is expressed in terms of the stream function $\eta$ defined such that $\mathbf{u}=\nabla \times \eta=(\partial \eta / \partial y,-\partial \eta / \partial x)^{T}$ so that the resulting synthetic velocity field is exactly divergence free. The two-point two-time correlation tensor of the turbulent velocity field $\mathbf{u}$, which is defined as $R_{i j}(\mathbf{x}, \mathbf{r}, t, s)=\left\langle u_{i}(\mathbf{x}, t) u_{j}(\mathbf{x}+\mathbf{r}, t+s)\right\rangle$ where $\langle\cdot\rangle$ denotes the ensemble average, can be related to the statistics of the stream function. To that end we also introduce the correlation of the stream function $C(\mathbf{x}, \mathbf{r}, t, s)=\langle\eta(\mathbf{x}, t) \eta(\mathbf{x}+\mathbf{r}, t+s)\rangle$.

Following Careta's et al. [10], and assuming homogeneous isotropic turbulence ${ }^{1}$, it is possible to relate $C$ with the radial correlation function $R(r)=R_{i i}(r, 0) / 2$. By using the property of isotropy in two dimensions, the Fourier transform of a turbulence statistic can be expressed in terms of the Bessel function of zeroth order $\mathrm{J}_{0}$. For instance, the correlation function $C$ and its Fourier transform $\hat{C}$ can be related by

$$
C(r)=\frac{1}{2 \pi} \int_{0}^{\infty} \hat{C}(\kappa) \mathrm{J}_{0}(\kappa r) \kappa \mathrm{d} \kappa, \quad \hat{C}(\kappa)=2 \pi \int_{0}^{\infty} C(r) \mathrm{J}_{0}(\kappa r) r \mathrm{~d} r,
$$

where $r=|\mathbf{r}|$ and $\kappa=|\boldsymbol{\kappa}|$. After combining equations (1) with the definition $\mathbf{u}=\nabla \times \eta$ and using properties of the Bessel functions, it is possible to obtain $\hat{R}(\kappa)=\kappa^{2} \hat{C}(\kappa) / 2$ which leads to the following expression

$$
R(r)=\frac{1}{2} R_{i i}(r, 0)=\frac{1}{4 \pi} \int_{0}^{\infty} \kappa^{3} \hat{C}(\kappa) \mathrm{J}_{0}(\kappa r) \mathrm{d} \kappa,
$$

which relates the radial correlation of velocity and the correlation of the stream function. Similarly, it is possible to relate the correlation of the stream function to the energy spectrum $E(\kappa)$ of the turbulence by using its definition in terms of the velocity spectrum [20]:

$$
E(\kappa)=\frac{1}{4 \pi} \kappa^{3} \hat{C}(\kappa) .
$$

We now introduce the stochastic model itself, which involves filtering a stochastic field to synthesize a random stream function featuring the required temporal and spatial correlation length. A two-dimensional turbulent flow can be achieved when the stream function $\eta$ is given by a convolution product [21]

$$
\eta(\mathbf{x}, t)=\int_{\mathbb{R}^{2}} G\left(\mathbf{x}-\mathbf{x}^{\prime}\right) U\left(\mathbf{x}^{\prime}, t\right) \mathrm{d} \mathbf{x}^{\prime}, \quad \mathbf{u}(\mathbf{x}, t)=\int_{\mathbb{R}^{2}} \mathbf{G}\left(\mathbf{x}-\mathbf{x}^{\prime}\right) U\left(\mathbf{x}^{\prime}, t\right) \mathrm{d} \mathbf{x}^{\prime},
$$

with $\mathbf{G}=\nabla \times G$.

The spatial correlation of the synthetic turbulence is controlled by the filter $G$ while the random field $U$ controls the temporal properties of the flow (the latter will be described in details in section 2.4). For small spatial and temporal separations, for which Taylor's hypothesis holds, $U$ satisfies the following properties:

$$
\langle U(\mathbf{x}, t)\rangle=0, \quad\langle U(\mathbf{x}, t) U(\mathbf{x}+\mathbf{r}, t+s)\rangle=\delta\left(\mathbf{r}-s \mathbf{u}_{0}\right) R_{U}(s),
$$

where $\mathbf{u}_{0}$ is the local convection velocity of the turbulence in the vicinity of $\mathbf{x}$ and $R_{U}(s)=\langle U(\mathbf{x}, t) U(\mathbf{x}, t+s)\rangle$ is the temporal correlation of $U$. Using the definition (4) together with a frame of reference moving with the mean flow $\mathbf{u}_{0}$, one can easily express the stream function correlation in terms of the filter:

$$
C(r, s)=(G * G)(r) R_{U}(s), \quad \hat{C}(\kappa, s)=\hat{G}(\kappa)^{2} R_{U}(s),
$$

where $*$ represents the convolution operator. Using these expressions together with equations (2) and (3) it is possible to relate the Fourier transform $\hat{G}$ of the filter to either the radial correlation or the energy spectrum:

$$
\hat{R}(\kappa)=\frac{1}{2} \kappa^{2} \hat{G}(\kappa)^{2}, \quad E(\kappa)=\frac{1}{4 \pi} \kappa^{3} \hat{G}(\kappa)^{2} .
$$

\footnotetext{
${ }^{1}$ In the present work we will consider homogeneous turbulence, but the theoretical results presented here are equally valid for locally homogeneous turbulence, that is to say when the integral length scale is much smaller than the length scale over which the turbulence statistics are varying. The method can in fact be extended to include strongly inhomogeneous turbulence [19].
} 
This yields two distinct definitions for the filter:

$$
G(r)=\frac{1}{\sqrt{2} \pi} \int_{0}^{\infty} \hat{R}(\kappa)^{1 / 2} \mathrm{~J}_{0}(\kappa r) \mathrm{d} \kappa, \quad G(r)=\frac{1}{\sqrt{\pi}} \int_{0}^{\infty}\left[\frac{E(\kappa)}{\kappa}\right]^{1 / 2} \mathrm{~J}_{0}(\kappa r) \mathrm{d} \kappa .
$$

Therefore one can choose to specify either the correlation or the energy spectrum, and it then follows a unique definition of the filter used to synthesize the turbulent velocity field. In this paper we use the latter option and we consider different filters based on three different choices for the turbulence energy spectrum.

\subsection{Application to different energy spectra}

In the context of aero-acoustic, stochastic methods based on filtering have relied mainly on Gaussian turbulence spectra but the method can also accommodate non-Gaussian spectra. One way to achieve this, as suggested by Siefert and Ewert [16], is to superimpose a series of Gaussian turbulent fields, each with a different correlation length and a different kinetic energy defined so as to obtain an arbitrary spectrum for the overall turbulence field. But this comes at the price of a significant increase in the computational cost. In this work we explore an alternative approach which is to use non-Gaussian filters that can achieve directly the specified energy spectrum. We present the method in this section and we will show in section 4 that it can indeed represent accurately non-Gaussian spectra.

The Gaussian spectrum originally proposed by Kraichnan [1] for synthetic turbulence only provides a basic description of the energy containing range and does not exhibit the expected algebraic rate of decay in the inertial sub-range. We will also consider the Liepmann spectrum which provides a better description of the energy containing range, as well as the von Kármán spectrum which provides a good description of both the energy containing range and the inertial sub-range. In two dimensions, these turbulence spectra are defined by

$$
E_{g}(\kappa)=\frac{2}{\pi^{2}} K \lambda^{4} \kappa^{3} \exp \left(-\frac{\lambda^{2} \kappa^{2}}{\pi}\right), \quad E_{l}(\kappa)=\frac{16}{3 \pi} K \lambda^{5} \frac{\kappa^{4}}{\left(1+\lambda^{2} \kappa^{2}\right)^{3}}, \quad E_{k}(\kappa)=\frac{110}{27 \pi} K \lambda \varsigma^{4} \frac{\kappa^{4}}{\left(1+\varsigma^{2} \kappa^{2}\right)^{17 / 6}},
$$

respectively. Here $K$ is the two-dimensional kinetic energy, $\lambda$ the integral length scale of the turbulence and $\varsigma=$ $\lambda \Gamma(1 / 3) / \sqrt{\pi} \Gamma(5 / 6)$ where $\Gamma$ is the Gamma function. These spectra are illustrated in figure 1.

When using these definitions of the energy spectra in the right-hand side of equation (8b), one can obtain closedform expressions for the corresponding filters:

$$
\begin{aligned}
G_{g}(r) & =\sqrt{\frac{2 K}{\pi}} \exp \left(-\frac{\pi r^{2}}{2 \lambda^{2}}\right), \\
G_{l}(r) & =\frac{4}{\pi} \sqrt{\frac{K}{3}}\left[\frac{\Gamma(1 / 4) \Gamma(5 / 4)}{\sqrt{\pi}}{ }_{1} \mathrm{~F}_{2}\left(\frac{5}{4} ; \frac{3}{4}, 1 ; \frac{r^{2}}{4 \lambda^{2}}\right)-\frac{\Gamma(3 / 4)}{\Gamma(5 / 4)} \sqrt{\frac{2 r}{\lambda}}{ }_{1} \mathrm{~F}_{2}\left(\frac{3}{2} ; \frac{5}{4}, \frac{5}{4} ; \frac{r^{2}}{4 \lambda^{2}}\right)\right], \\
G_{k}(r) & =\frac{1}{\pi} \sqrt{\frac{110 K \lambda}{3 \varsigma}}\left[\frac{\Gamma(7 / 6) \Gamma(5 / 4)}{\Gamma(17 / 12)}{ }_{1} \mathrm{~F}_{2}\left(\frac{5}{4} ; \frac{5}{6}, 1 ; \frac{r^{2}}{4 \varsigma^{2}}\right)-\frac{\Gamma(5 / 6)}{\Gamma(7 / 6)}\left(\frac{r}{2 \varsigma}\right)^{1 / 3}{ }_{1} \mathrm{~F}_{2}\left(\frac{17}{12} ; \frac{7}{6}, \frac{7}{6} ; \frac{r^{2}}{4 \varsigma^{2}}\right)\right],
\end{aligned}
$$

where ${ }_{1} \mathrm{~F}_{2}$ is the generalized hypergeometric function (see chapter 15 in [22]). A comparison of the filters derived from the Gaussian, Liepmann and von Kármán spectra in physical space is shown in figure 1.

At this stage, it is useful to comment on several features of these filters. First of all, the Gaussian filter has the interesting property that it is separable in space. This allows for efficient implementations of the filtering procedure where the filter is applied separately in each spatial directions (see [23] for more details on this feature). This is however to put in perspective with the poor description of the turbulence spectra provided by the Gaussian spectrum.

Secondly, the use of these filters involves calculating exponentials or hypergeometric functions which can be very costly, especially because for a typical simulation one has to evaluate these filters several million times. An attempt was therefore made in this work to use interpolated filters which are much faster than the exact expressions. It was found that very accurate interpolations of the three filters can be obtained using rational interpolants provided that two specific issues are taken into consideration. 
- All filters decay at infinity so one only needs to interpolate them up to a distance $(r / \lambda)_{\max }$ beyond which the contribution of the filter can be considered negligible. This distance is however larger for the Liepmann and von Kármán filters compared to the Gaussian filter, because for large distances $(r \rightarrow \infty)$ the Liepmann and von Kármán filters decay algebraically while the Gaussian filter decreases exponentially. This has practical consequences that will be discussed in section 3.3 .

- Using asymptotic expansions, it can be shown that the Gaussian filter has a regular behaviour $G_{g}(r) \approx G_{0}+G_{1} r^{2}$ near $r=0$ whereas the Liepmann and von Kármán filters behave as $G_{l}(r) \approx L_{0}+L_{1} r^{1 / 2}$ and $G_{k}(r) \approx K_{0}+K_{1} r^{1 / 3}$, respectively. This lack of smoothness near the origin for the Liepmann and von Kármán filters can be directly related to the algebraic rate of decay of the respective spectra for large wavenumbers [24, page 36]. The exponential decay of the Gaussian spectrum leads to a smooth behaviour of the filter near $r=0$. When building interpolations of the Liepmann and von Kármán filters it is important to ensure that this behaviour is accurately captured.

In this work, rational interpolations of fourth and fifth order have been used for the three filters. Results in sections 4 and 5 show that very accurate results can be obtained despite the use of interpolation. For the Liepmann and von Kármán spectra, the use of interpolated filters results in speedups of the order of 12000 or 15000 compared to evaluating these filters exactly. The use of interpolation is therefore crucial to achieving good performance.

\subsection{Vortex particles}

To obtain a numerical method describing the turbulent field in terms of random vortex particles the filtering equation (4) is now discretized using a fully Lagrangian formulation and following an approach similar to reference [15]. To that end, we denote $\mathbf{y}\left(\mathbf{r}_{0}, t\right)$ the trajectory of the fluid element located at $\mathbf{r}_{0}$ at the initial time $t_{0}$. And we can define the initial volume of fluid containing the turbulent field by $\Omega_{0}$ at $t_{0}$. The Jacobian of the transformation from Eulerian coordinates $\mathbf{y}$ to Lagrangian coordinates $\mathbf{r}_{0}$ is written $J=\left|\mathrm{d} \mathbf{y} / \mathrm{d} \mathbf{r}_{0}\right|$. Note that for an incompressible flow we have $J=1$. Using this notation, the convolution integral (4) can be written using Lagrangian coordinates:

$$
\mathbf{u}(\mathbf{x}, t)=\int_{\Omega_{0}} \mathbf{G}\left(\mathbf{x}-\mathbf{y}\left(\mathbf{r}_{0}, t\right)\right) U\left(\mathbf{r}_{0}, t\right) J \mathrm{~d} \mathbf{r}_{0} .
$$

Then by discretizing the volume $\Omega_{0}$ into a set of non-overlapping elements $\left\{\Omega_{0 n}\right\}_{n=1}^{N}$ one can write:

$$
\mathbf{u}(\mathbf{x}, t)=\sum_{n=1}^{N} \int_{\Omega_{0 n}} \mathbf{G}\left(\mathbf{x}-\mathbf{y}\left(\mathbf{r}_{0}, t\right)\right) U\left(\mathbf{r}_{0}, t\right) J \mathrm{~d} \mathbf{r}_{0},
$$

Each element $\Omega_{0 n}$ can be understood as a small fluid element moving through the computational domain and whose trajectory $\mathbf{r}_{n}(t)$ is defined as the centre of mass of $\Omega_{n}$ :

$$
\mathbf{r}_{n}(t)=\int_{\Omega_{0 n}} \mathbf{y}\left(\mathbf{r}_{0}, t\right) J \mathrm{~d} \mathbf{r}_{0}
$$

If we now assume that the fluid elements are sufficiently small compared to the integral length scale $\lambda$, so that it is possible to consider that $\mathbf{G}$ is almost constant over each element, then we can arrive at

$$
\mathbf{u}(\mathbf{x}, t)=\sum_{n=1}^{N} \mathbf{G}\left(\mathbf{x}-\mathbf{r}_{n}(t)\right) U_{n}(t),
$$

where $U_{n}$ is the weighted average of $U$ over a fluid element:

$$
U_{n}(t)=\int_{\Omega_{0 n}} U\left(\mathbf{r}_{0}, t\right) J \mathrm{~d} \mathbf{r}_{0}
$$

Equation (13) is the central definition of the random particle method. It shows that the synthetic turbulent velocity field can be understood as the sum of $N$ vortices with positions $\mathbf{r}_{n}(t)$ and random strengths $U_{n}(t)$. The velocity induced by 
each vortex is defined by the vector field $\mathbf{G}$ which follows directly from the choice of an energy spectrum, as explained in section 2.2.

In the present work the vortices are simply convected by a given mean flow $\mathbf{u}_{0}(\mathbf{x})$ which amounts to solve $\mathrm{d} \mathbf{r}_{n} / \mathrm{d} t=$ $\mathbf{u}_{0}\left(\mathbf{r}_{n}\right)$. Interactions between vortices are therefore neglected. This is in contrast with more standard vortex particle methods where computing the interactions between vortices is a central aspect of the solution procedure [25]. Note that interaction between vortices could also be included in the RPM [16].

The velocity field can be computed directly using (13) where the contributions of all the vortices are summed at any point $\mathbf{x}$ where the synthetic velocity field is needed. An alternative is to use an auxiliary grid for the filtering of the stochastic field $U$. In that case the strength $U_{n}$ of the vortex particles are projected onto this auxiliary grid which is then used to apply the filtering (4) and to compute the curl of the stream function. As shown by Ewert et al. [11], this is useful when one uses the Gaussian filter since one can apply the filtering in each spatial direction independently.

\subsection{Temporal properties of the synthetic turbulence}

We now concentrate on defining the stochastic processes used to compute the random vortex strengths $U_{n}(t)$. These processes control the temporal properties of the synthetic turbulence field. Several levels of sophistication will be considered here, from the simplest model of frozen turbulence to two models representing evolving turbulence, characterized by either one or two time scale(s).

\subsubsection{Frozen turbulence}

If we assume that the velocity field seen by an observer moving with the base flow is constant (hence the name 'frozen' turbulence) then for this observer the random field $U$ is also independent of time. If we assume that the mean flow is incompressible $(J=1)$ then it follows from (14) that the vortex strengths is constant in time: $U_{n}(t)=U_{n}(0)$. The initial condition is a Gaussian random variable with zero mean and a variance equal to the area of $\Omega_{n}$. In that case it can be shown that the time correlation defined by (5) is simply $R_{U}(s)=1$, indicating that apart from the convection effect there is no loss of correlation in time.

In the case of a compressible mean flow, $J$ varies in time, and one has to scale the strength $U_{n}(t)$ such that its variance is given by

$$
\sigma_{n}(t)=\int_{\Omega_{0 n}} J^{2} \mathrm{~d} \mathbf{r}_{0} .
$$

\subsubsection{Evolving turbulence: a single Langevin equation}

Frozen turbulence essentially assumes that the integral time scale of the turbulence is infinite. A more accurate representation of the turbulence field can be obtained by including the effects of a finite integral time scale $T_{L}$. This can be achieved using Langevin equations which are stochastic differential equations originally devised to represent Brownian motion and now commonly used to describe turbulent diffusion at large Reynolds numbers. The strength of each vortex is evolved in time by solving the following Langevin equation: ${ }^{2}$

$$
\mathrm{d} U_{n}=-\alpha U_{n} \mathrm{~d} t+\beta \mathrm{d} W_{n}
$$

with initial condition $U_{n}(0)=U_{n 0}$ which is also a random variable following the same distribution as in the previous section. The second term on the right-hand side (the diffusion term) involves a Wiener process $W_{n}$ (for a complete definition of a Wiener process the reader can refer to [26]). The coefficients $\alpha$ and $\beta$ of the Langevin equation can be related to the statistical properties of the turbulence. First of all the requirement that the process $U_{n}(t)$ be statistically stationary yields $\beta=\left(2 \alpha\left\langle U_{n 0}^{2}\right\rangle\right)^{1 / 2}$. It then follows that the correlation of the random process is

$$
\left\langle U_{n}(t) U_{n}(t+s)\right\rangle=\left\langle U_{n 0}^{2}\right\rangle e^{-\alpha|s|},
$$

which leads to the conclusion that $\alpha=1 / T_{L}$. With this model the correlation $R_{U}(s)$ defined in equation (5) is exponential $R_{U}(s)=\left\langle U_{0}^{2}\right\rangle e^{-|s| / T_{L}}$. In fact, when assuming frozen turbulence, the integral time scale tends to infinity,

\footnotetext{
${ }^{2}$ Here we will follow the standard notations for stochastic differential equations where differentials are used in favour of derivatives (see for instance chapter 3 in [26]).
} 
$\alpha \rightarrow 0$, and hence the right-hand side of (15) vanishes, meaning that the vortex strengths remain constant. Note also that there is technically no requirement to use the same time scale $T_{L}$ and intensity $\left\langle U_{n 0}^{2}\right\rangle$ for all the vortices, and each vortex could have different properties.

The subscript $L$ indicates that $T_{L}$ is the Lagrangian integral time scale. In practice it is often easier to define Eulerian time scales, but it is possible to relate the Eulerian and Lagrangian time scales. For instance $T_{L}$ can be estimated using the following scaling argument [27]:

$$
T_{L} \approx \frac{2 K}{C_{0} \epsilon}
$$

where $\epsilon$ is the dissipation rate and $C_{0}$ an empirical constant which is understood to be in the range 1 to 6 . In this work we use $C_{0}=2.1$ as proposed by Pope in [27].

The Langevin equation (15) can be discretized using a simple forward Euler scheme [26, chapter 9]:

$$
U_{n}(t+\Delta t)=(1-\alpha \Delta t) U_{n}(t)+\beta \sqrt{\Delta t} N_{n}(t),
$$

where $N_{n}(t) \in \mathcal{N}(0,1)$ are white noise signals that can be generated using any standard random number generators.

\subsubsection{Evolving turbulence: a second-order Langevin model}

The presence of the Wiener process $W_{n}$ in the second term on the right-hand side of equation (15) implies that the resulting stochastic process $U_{n}(t)$ is continuous but not differentiable in time, see [26]. As will be illustrated in section 5.1 this lack of smoothness introduces spurious sources of noise in the numerical simulations of the acoustic field. To overcome this problem, a second-order Langevin equation is also considered. Although the general idea used here is similar to what was briefly outlined in Siefert and Ewert [16], we follow the analysis of Krasnoff et al. [28] which has the advantage of also providing a physical interpretation of the method.

The idea proposed in reference [28] is to use instead of white noise a forcing term in the Langevin equation that features its own time scale:

$$
\mathrm{d} U_{n}=-\alpha U_{n} \mathrm{~d} t+V_{n} \mathrm{~d} t
$$

with initial condition $U_{n}(0)=U_{n 0}$ where $U_{n 0}$ is a random variable following a Gaussian distribution with zero mean and variance $\sigma_{n}$. In contrast with the diffusion term $\mathrm{d} W_{n}$ in the Langevin equation (15), the diffusion term $V_{n}$ is now assumed to be continuous (so it is not pure white noise) and it is also expected to be correlated with $U_{n}$. To fix ideas we will consider an exponential correlation for the process $V_{n}$ :

$$
R_{V}(s)=\left\langle V_{n}(t) V_{n}(t+s)\right\rangle=\left\langle V_{n 0}^{2}\right\rangle e^{-\gamma|s|},
$$

with $\gamma>0$. From the requirement that the random process $U_{n}(t)$ is statistically stationary, one can derive

$$
\left\langle U_{n 0} V_{n}(t)\right\rangle=\alpha\left\langle U_{n 0}^{2}\right\rangle e^{\alpha t}\left[1-\frac{1}{\alpha\left\langle U_{0}^{2}\right\rangle} \int_{0}^{t} e^{-\alpha s} R_{V}(s) \mathrm{d} s\right] .
$$

It is reasonable to assume that the correlation (20) between $U_{n 0}$ and $V_{n}(t)$ vanishes with time. It follows from the condition $\left\langle U_{n 0} V_{n}(t)\right\rangle \rightarrow 0$ as $t \rightarrow \infty$ that $\left\langle V_{n 0}^{2}\right\rangle=\alpha(\alpha+\gamma)\left\langle U_{n 0}^{2}\right\rangle$. This leads to

$$
\left\langle U_{n 0} V_{n}(t)\right\rangle=\alpha\left\langle U_{n 0}^{2}\right\rangle e^{-\gamma t},
$$

and the resulting time correlation for $U_{n}(t)$ is

$$
R_{U}(s)=\left\langle U_{0}^{2}\right\rangle \frac{\gamma e^{-\alpha|s|}-\alpha e^{-\gamma|s|}}{\gamma-\alpha}=\left\langle U_{n 0}^{2}\right\rangle e^{-\alpha|s|}\left\{1+\frac{\alpha}{\gamma-\alpha}\left[e^{-(\gamma-\alpha)|s|}-1\right]\right\} .
$$

As we have explained, the Langevin model (18) includes two time scales, namely the time scale $1 / \gamma$ of the diffusion term and the time scale $1 / \alpha$ of the drift term. Alternatively, it is possible from equation (22) to define an integral time scale $T_{L}$ and a micro time scale $\tau_{L}$ as follows:

$$
T_{L}=\int_{0}^{\infty} R_{U}(s) \mathrm{d} s=\frac{1}{\alpha}+\frac{1}{\gamma}, \quad \tau_{L}=\sqrt{\frac{2}{\alpha \gamma}} .
$$


The latter is identified by noting that for small time separation $s \rightarrow 0$ we have $R_{U}(s) /\left\langle U_{n 0}^{2}\right\rangle \approx 1-\gamma \alpha s^{2} / 2=1-\left(s / \tau_{L}\right)^{2}$. In addition it can be seen that when $\gamma$ is much larger than $\alpha$ one recovers the exponential correlation $\left\langle U_{n 0}^{2}\right\rangle e^{-\alpha|s|}$ modeled by the standard Langevin equation (15).

As suggested by Krasnoff et al. [28] a physical interpretation of the micro time scale $\tau_{L}$ is that it represents the viscous diffusion process. And $\tau_{L}$ can therefore be considered to be of the order of the Kolmogorov time scale. But this is not mandatory, and one can consider $\tau_{L}$ as a numerical parameter introduced primarily for computational reasons in order to smooth the synthetic velocity field. We will discuss in details in section 5 how this parameter can be chosen in relation with the time step and $T_{L}$.

The stochastic source $V_{n}$ in the Langevin equation in (18) is now fully defined and it can be generated using a second Langevin equation similar to (15):

$$
\mathrm{d} V_{n}=-\gamma V_{n} \mathrm{~d} t+\sqrt{2 \gamma\left\langle V_{n 0}^{2}\right\rangle} \mathrm{d} W_{n},
$$

where the diffusion term has been chosen such that the process $V_{n}(t)$ is statistically stationary. From equation (21) it appears that the initial condition $V_{n}(0)=V_{n 0}$ is in fact correlated with $U_{n 0}$ so that $\left\langle U_{n 0} V_{n 0}\right\rangle=\alpha\left\langle U_{n 0}^{2}\right\rangle$. It can be generated for instance by using: ${ }^{3}$

$$
V_{n 0}=\alpha U_{n 0}+\sqrt{\alpha \gamma} \xi,
$$

where $\xi$ is an independent Gaussian random variable with zero mean and variance $\sigma_{n}$. The coefficient $\sqrt{\alpha \gamma}$ is required to satisfy $\left\langle V_{n 0}^{2}\right\rangle=\alpha(\alpha+\gamma)\left\langle U_{n 0}^{2}\right\rangle$. [26]:

When discretized using a forward Euler scheme, the system of coupled Langevin equations (18) and (24) becomes

$$
\left\{\begin{array}{l}
U_{n}(t+\Delta t)=(1-\alpha \Delta t) U_{n}(t)+\Delta t V_{n}(t), \\
V_{n}(t+\Delta t)=(1-\gamma \Delta t) V_{n}(t)+\sqrt{2 \gamma \Delta t\left\langle V_{n 0}^{2}\right\rangle} N_{n}(t),
\end{array}\right.
$$

where the time series $N_{n}(t) \in \mathcal{N}(0,1)$ are sampled using Gaussian random number generators.

\section{Broadband fan interaction noise}

We now validate the random particle method using broadband fan interaction noise as a target application. To that end we use the linearized Euler equations in two dimensions as a propagation model solved in the time domain. As a benchmark problem, we consider the broadband noise generated by the interaction between isotropic homogeneous turbulence and a flat plate with zero angle of attack.

\subsection{Linearized Euler equations}

The unsteady field around the airfoil is modeled using the Linearized Euler Equations (LEE). Assuming that the fluctuations are isentropic and small compared to the mean flow, the acoustic variables are given by

$$
\mathbf{u}=\mathbf{u}_{0}+\mathbf{u}^{\prime}, \quad p=p_{0}+p^{\prime}, \quad \rho=\rho_{0}+\rho^{\prime},
$$

where $\mathbf{u}$ is the velocity field, $\rho$ the density and $p$ the pressure. Subscripts 0 denote mean values and ' denotes fluctuating components. The two-dimensional linearized Euler equations for a uniform mean flow can be written

$$
\frac{\partial \mathbf{q}}{\partial t}+\frac{\partial \mathbf{A q}}{\partial x}+\frac{\partial \mathbf{B q}}{\partial y}=\mathbf{0}
$$

with the following definitions

$$
\mathbf{q}=\left[\begin{array}{c}
\rho^{\prime} \\
(\rho u)^{\prime} \\
(\rho v)^{\prime} \\
p^{\prime}
\end{array}\right], \quad \mathbf{A}=\left[\begin{array}{cccc}
0 & 1 & 0 & 0 \\
-u_{0}^{2} & 2 u_{0} & 0 & 1 \\
-u_{0} v_{0} & v_{0} & u_{0} & 0 \\
-u_{0} c_{0}^{2} & c_{0}^{2} & 0 & u_{0}
\end{array}\right], \quad \mathbf{B}=\left[\begin{array}{cccc}
0 & 0 & 1 & 0 \\
-u_{0} v_{0} & v_{0} & u_{0} & 0 \\
-v_{0}^{2} & 0 & 2 v_{0} & 1 \\
-v_{0} c_{0}^{2} & 0 & c_{0}^{2} & v_{0}
\end{array}\right]
$$

\footnotetext{
${ }^{3}$ Alternatively, one could use an independent random variable for $V_{n 0}$ and generate a correlated initial value for $U_{n}(t)$.
} 
where $c_{0}$ is the mean sound speed.

These equations are solved in the time domain using a parallel, multi-block, finite-difference solver. It relies on seven-point dispersion-relation-preserving stencils for spatial derivatives $[29,30]$ and an optimized six-stage RungeKutta scheme for the time integration [31]. Non-reflecting boundary conditions are applied at the boundaries of the computational domain [32]. Also implemented is a selective filter that removes short wavelength components that are poorly resolved by the grid [31]. Finally, acoustic results in the far field are computed with the Ffowcs Williams Hawkings equation.

\subsection{Coupling with the Synthetic Turbulence}

The unsteady disturbances propagating on the mean flow are composed of the incident turbulent velocity field and the scattered field induced by the interaction of the airfoil with the turbulence. The incident turbulence is generated by the vortex particle method. The linearized Euler equations are solved for the scattered field only which is comprised of the radiated sound but also of the vorticity shed at the airfoil trailing edge. The coupling between incident and scattered fields occurs along the airfoil where the total normal velocity should vanish, implying that $\mathbf{u}^{\prime} \cdot \mathbf{n}=-\mathbf{u}_{t} \cdot \mathbf{n}$ where $\mathbf{u}_{t}$ is the incident turbulent velocity. This boundary condition is implemented in the finite-difference LEE solver by updating the solution along the airfoil using the characteristics formulation of Kim et al. [33].

It should be noted that if one uses a RANS simulation to characterize the turbulent velocity field $\mathbf{u}_{t}$ to impose on the airfoil, then the simulation will invariably yield a zero kinetic energy along the airfoil surface. This could be addressed by computing the velocity field induced by the vorticity field as if in free field, that is by ignoring the presence of the solid surface of the airfoil. ${ }^{4}$

\subsection{Computational Setup}

The test case considered here is that of a flat plate with zero angle of attack interacting with homogeneous isotropic turbulence convected by a uniform mean flow. The problem is made non-dimensional using the chord $c$, mean density $\rho_{0}$, and sound speed $c_{0}$.

The computational setup is shown in figure 2. The simulation domain is a rectangle of size $3 c \times 2 c$ centered on the airfoil. A Cartesian grid is used with $1200 \times 800$ grid points. The time step is such that the CFL number is 0.8 .

A closed Ffowcs Williams - Hawkings control surface is placed around the airfoil. Thompson's multitaper method [34] was used to compute the numerical power spectral density using as input the time-domain signal obtained as the Fourier transform of the Ffowcs Williams - Hawkings formulation. The frequency range of interest was found to correspond to Strouhal numbers $S t=f c / u_{0}$ between 0 to 5 .

For the synthetic turbulence, vortex particles are distributed over a rectangular region around the airfoil. The size of this vortex region is based on the distance $r_{\max }$ defined in section 2.2 since the contribution of any vortex farther than $r_{\max }$ from the airfoil is negligible. Vortex particles are created at the upstream boundary of the vortex region, they are then convected downstream with the mean flow and finally removed from the simulation once they reach the other end of the vortex region.

For the case of frozen turbulence the strength of each vortex particle remains constant and is randomly generated using a Gaussian distribution defined in section 2.4.1. For evolving turbulence, the strength of the vortices is updated every stage of the Runge-Kutta method as defined by either (17) or (25) depending on the Langevin model under consideration.

An extensive series of parametric studies was conducted for all three filters in order to adjust the distribution of particles in the vortex region (the details are available in [20]). It was found that to obtain accurate results the spacing $\delta$ between vortices can be chosen as $\delta=\lambda / 6, \lambda / 10$ and $\lambda / 10$ for the Gaussian, Liepmann and von Kármán spectra, respectively. The higher density of vortex particles required for the Liepmann and von Kármán spectra is due to the larger energy content seen for large wavenumbers.

\footnotetext{
${ }^{4}$ This issue was kindly pointed out by one of the reviewers.
} 


\section{Results for frozen turbulence}

Firstly, the accuracy of correlations and energy spectra of the synthetic velocity field is assessed together with the scattered acoustic pressure in the near and far field assuming frozen turbulence. Then in the next section, the effects of time correlation will be included in the model to generate synthetic turbulence.

The parameters of the benchmark problem are similar to the test case considered by Amiet in [18]. The turbulence is convected by a uniform mean flow with Mach number 0.362, the turbulent integral length scale is $\lambda=0.07$.

\subsection{Synthetic turbulence}

The quality of the synthetic turbulence generated by the random particle method has been assessed in terms of the two-point correlations $R_{11}\left(r \mathbf{e}_{1}, 0\right)$ and $R_{22}\left(r \mathbf{e}_{1}, 0\right)$ along the airfoil and also in terms of the one-dimensional spectra $E_{11}(\kappa)$ and $E_{22}(\kappa)$ where $E_{i j}(\kappa)$ is defined as the Fourier transform of $2 R_{i j}(r)$. These results are shown in figures 3 to 5 for each of the three energy spectra considered here. In all cases there is very good agreement between the numerical results and the theoretical definitions of the correlation and one-dimensional energy spectrum, demonstrating that the random particle method is able to capture these properties accurately. For the correlation functions only minute deviations from the expected results can be observed. These are more clearly visible in the one-dimensional energy spectra where they appear at high frequencies.

It was also found that the statistical variance of the spectra obtained from the numerical simulations tends to increase at high frequencies, as can be seen in figures 4 and 5. This is not visible nor relevant for the Gaussian spectrum due to its exponential decay. For the Liepmann and von Kármán spectra it is therefore preferable to acquire longer time series so as to reduce the variance of the predicted spectrum at high frequencies.

\subsection{Acoustic pressure}

Figure 6 shows a snapshot of the acoustic pressure in the near field around the airfoil for the Gaussian spectrum. As expected, the noise is radiated from the leading edge of the airfoil in the form of a dipole. The sound waves propagate along the airfoil and are then back-scattered at the trailing edge.

The predicted sound power levels (SPL) computed in the far field has been compared against the analytical solution derived by Amiet [18] in terms of both noise spectrum and directivity. Note that this analytical solution has been modified to account for a fully two-dimensional problem [20]. The sound power levels presented in this work are normalized by the distance between the observer and the center of the flat plate.

Figure 7 shows the numerical and theoretical sound pressure levels obtained with the Gaussian spectrum for observers located at 30,60,120, and 150 degrees (angles are measured from the downstream direction). The corresponding results obtained with the Liepmann and von Kármán spectra are given in figures 8 and 9. Overall, noise levels are in very good agreement with the analytical solution for the three spectra at all locations. The only significant discrepancies are found for the Liepmann and von Kármán spectra in the upstream direction for Strouhal numbers above 3, where the numerical results tend to over-predict the troughs in the SPL. Note however that these discrepancies are only observed when the noise levels are more than $15 \mathrm{~dB}$ below the main peak of noise.

Far-field directivities for Strouhal numbers $S t=2.03$ and $S t=4.06$ are shown in figure 10 for the Gaussian spectrum. Very good agreement is obtained at all angles, although the error increases slightly at large angles. Similar levels of accuracy are obtained for Liepmann and von Kármán spectra, due to the fact that the turbulence spectrum only affects the absolute level of the directivity at any given frequency.

\section{Results for evolving turbulence}

We now turn to the case of evolving turbulence by introducing a finite correlation time. First we use the standard Langevin equation (17) to model the temporal decorrelation. Then we demonstrate how the second-order Langevin model (25) avoids the generation of spurious noise sources. To simplify the discussion and focus on the effects of evolving turbulence, simulation results will only be presented for the Gaussian energy spectrum. 


\subsection{Standard Langevin equation}

The validation of the evolving synthetic turbulence with integral time scale $T_{L}$ is performed by comparing numerical and theoretical results for the two-point space-time correlation $R_{i j}(r, t)=\left\langle u_{i}^{\prime}\left(r_{1}, t_{1}\right) u_{j}^{\prime}\left(r_{2}, t_{2}\right)\right\rangle$ along the flat plate with different spatial separations, as shown in figure 11. Very good agreement is observed between the numerical and analytical correlations $R_{11}$ and $R_{22}$ at all locations. In addition, the temporal decorrelation introduced by the Langevin equation (17) is accurately described with the expected exponential decay $\exp \left(-t / T_{L}\right)$. This illustrates that the standard Langevin equation (17) is able to capture all the required features of the turbulent velocity correlation.

However, for the radiated sound field the situation is quite different. The acoustic pressure in the near field is shown in figure 12a. When compared with the acoustic near field observed with frozen turbulence (see figure 6), it is clear that the radiated noise has been altered significantly. In addition to the noise radiated from the leading edge of the airfoil, additional noise sources appear to be distributed along the airfoil and to radiate in the vertical direction. The amplitude of the noise propagating upstream has also increased significantly.

These observations are confirmed by the far-field results. Figure 13 shows the normalized sound pressure spectrum calculated using the standard Langevin model (17) compared against the numerical results for frozen turbulence. Also included is an extension of Amiet's analytical solution [18] to include the effects of the exponential temporal correlation $\exp \left(-|s| / T_{L}\right)$. It is worth noting that the effect of $T_{L}$ is small in this case and that the analytical results with frozen or evolving turbulence are in fact very similar. The far-field directivity is presented in figure 14 for two frequencies. These results show more quantitatively that the evolving turbulence model (6) results in a large increase of sound pressure levels at high-frequencies and especially in the upstream direction. Noise levels at 120 and 150 degrees are larger for evolving turbulence than those predicted by the analytical model over the whole range of frequencies. In particular, at 150 degrees an almost flat spectrum is found for Strouhal numbers larger than 2. At 150 degrees, the interference pattern originally generated by the interaction between the noise radiated from the leading edge and the scattering at the trailing edge is no longer present. In addition, sound pressure levels at $S t=4.06$ are larger upstream than downstream.

A lack of numerical resolution in space or time has been ruled out as a potential explanation for the changes observed in the previous section. The smallest hydrodynamic wavelengths are resolved by 17 points per wavelength and the smallest acoustic wavelengths by 35 points per wavelength in the frequency range of interest ( $S t=0$ to 5 ). In addition, using a smaller time step produced almost identical results.

The root cause for this issue can be found in the use of the Langevin equation (17) within a high-order finitedifference method solving the linearized Euler equations, and more specifically in the lack of smoothness in time of the synthetic velocity field generated by (17). This can be seen more clearly by noting that it is the material derivative $\mathrm{d}_{0} v_{n} / \mathrm{d} t$ of the imposed normal velocity $v_{n}$ which acts as a dipole source along the surface of the airfoil. If we rewrite this term in a frame of reference moving with the mean flow and use (13) we get

$$
\frac{\partial v_{n}}{\partial t}=\mathbf{n} \cdot \sum_{n} \mathbf{G}\left(\mathbf{x}-\mathbf{r}_{n}\right) \frac{\mathrm{d} U_{n}}{\mathrm{~d} t}=\mathbf{n} \cdot \sum_{n} \mathbf{G}\left(\mathbf{x}-\mathbf{r}_{n}\right)\left(-\alpha U_{n}+\beta \frac{\mathrm{d} W_{n}}{\mathrm{~d} t}\right),
$$

where the derivative $\mathrm{d} W_{n} / \mathrm{d} t$ can be understood as a white noise signal. This indicates that the use of the standard Langevin equation amounts to introduce a white noise source in the numerical model of the linearized Euler equations. This has several consequences:

- The introduction of white noise source is an issue for the stability of the time integration scheme since it can only resolve fluctuations up to a certain frequency. And even if the numerical integration is not unstable the accuracy of the results could be questioned. Note that this applies solely to the time-integration of the LEE using a Runge-Kutta scheme and is not related with the integration of the Langevin equations themselves. The latter are integrated using an Euler scheme which is stable for this type of stochastic equations.

- This white noise source contributes equally at all frequencies and is therefore responsible for the relatively flat spectrum that seems to dominate at high frequencies in some of the graphs in figure 13. The issue therefore is that for these high frequencies the spectrum is not representative of the actual turbulence spectrum but is only a consequence of the numerical implementation of the stochastic model and of the choice of a white noise source term in (17) 
One way to address these issues could be to use Runge-Kutta schemes modified specifically for stochastic differential equations, see [26]. Such time integration scheme would be able to solve more accurately for the white noise source found in (28), but that would require applying this scheme only for the grid points on the airfoil. This approach would be needed if using the standard Langevin equation is required.

Another option was presented in section 2.4.3, and consists in modeling the time correlation of the turbulence in such a way that the time derivative synthetic velocity field is smoother, which is equivalent to removing only the high-frequency content. Note that with this approach the correlation function is (22) rather than $\exp \left(-|s| / T_{L}\right)$. But, as explained above, in practice the differences are very small.

\subsection{Second-order Langevin model}

The second-order Langevin model described in section 2.4.3 introduces an additional small time scale $\tau_{d}$. Its effect is illustrated in figure 15 where the evolution of a vortex-particle strength computed with the second-order Langevin model (25) for different values $\tau_{d}$ is compared against the results obtained with (17). The smoothing, or averaging, introduced by the small time scale $\tau_{d}$ is clearly visible, with the solution becoming smoother as $\tau_{d}$ increases. Figure 15 also illustrates that a large value of $\tau_{d}$ yields results that become very different from the original results.

When selected properly $\tau_{d}$ allows to obtain smooth results without modifying significantly the integral time scale of the synthetic turbulence. Firstly, $\tau_{d}$ must be sufficiently smaller than the integral time scale $T_{L}$ of the turbulence so that the resulting temporal decorrelation of the vortex strength (22), is a good approximation of $\exp \left(-t / T_{L}\right)$. Secondly, $\tau_{d}$ should be sufficiently large compared to the numerical time step in order to discretize (25). If the numerical time step is too large then the small time scale $\tau_{d}$ is not captured and one is essentially solving the standard Langevin equation (17).

A parametric study has been performed to adjust the value of $\tau_{d}$ [20] and it was concluded that by using $T_{L} / \tau_{d} \approx 70$ the temporal decorrelation of the turbulence is accurately captured by the model and convergent far-field results can be obtained using the same time step as for the case of frozen turbulence $\left(\tau_{d} / \Delta t \approx 100\right)$. It should be noted that with the second-order Langevin equation (25) the low-frequency fluctuations in $U_{n}(t)$ that characterize the integral time scale $T_{L}$ of the evolving turbulence remain unchanged while the high-frequency fluctuations that are causing the issues observed in the previous section are efficiently removed.

The acoustic near field obtained with these choices of numerical parameters is shown in figure 12b. As expected the high-frequency noise radiating form the middle of the flat plate predicted by the Langevin equation (17) and seen in figure $12 \mathrm{a}$ is no longer present.

Normalized sound pressure levels in the far field obtained when using the second-order Langevin model are shown in figure 16 and compared with the numerical results for frozen turbulence and the analytical solution for evolving turbulence. In contrast with the figure 13 one can see clearly that the spurious high-frequency noise has been removed. Similar sound pressure levels are obtained for the five locations either by assuming that the turbulence is frozen or by including the effects of time correlation through the second-order Langevin model. The only discrepancy found is at 150 degrees for evolving turbulence, sound pressure levels do not fully account for the interference pattern generated by interaction between the noise radiated from the leading edge and the scattering at the trailing edge.

Directivities at the Strouhal numbers $S t=2.03$ and $S t=4.06$ are shown in figure 17. For both Strouhal numbers, directivities predicted using evolving turbulence are similar to the case of frozen turbulence apart form upstream directions where slightly different trends are found. This disparity for upstream directions can be related with the discrepancy found in the frequency spectra at upstream locations, see figure 16.

\section{Conclusions}

A random particle method has been proposed to predict the broadband noise generated by the interaction of a turbulent stream with an airfoil. The RPM is able to capture efficiently and accurately the two-point two-time correlation as well as the turbulence energy spectra. To describe aerodynamic noise generation and radiation the RPM was coupled with a finite-difference solver for the linearized Euler equations. The complete approach was validated against Amiet's model for the broadband noise generated by the interaction between a two-dimensional flat plate and homogeneous isotropic turbulence. The general conclusion is that the RPM is well suited for describing this kind of noise source mechanism. 
A novel feature of the RPM described in this paper is the use of non-Gaussian filter kernels to represent different energy spectra, including Liepmann and von Kármán spectra. It was demonstrated that this approach yields accurate results. And using interpolated filters, instead of their exact expressions, has proved critical to maintain computational efficiency without introducing significant errors.

Another aspect that was investigated in this paper is the description of temporal decorrelation. This was achieved two different stochastic equations for the vortex strengths. The first is a standard Langevin equation using a white noise field as forcing term (i.e. Wiener process). The statistical behaviour of the resulting evolving turbulent flow captures the expected exponential temporal decorrelation in time and reproduces two-point space-time correlations accurately. But the radiated sound field exhibits much larger amplitudes at high frequencies. This is not a genuine physical effect but a numerical issue introduced by the white noise diffusion term in the Langevin equation. A secondorder Langevin model was described to generate smoother synthetic velocity fields by using a forcing term that is continuous. The resulting stochastic model provides accurate two-point space-time correlations along the flat plate but also yields more reliable far-field noise predictions.

The introduction of time decorrelation leads to results quite similar to those obtained with frozen turbulence. This is confirmed by the analytical solution modified to include temporal decorrelation which has been used to validate the numerical results. This can be explained by the fact that the characteristic time scale of a vortex moving past the sharp leading edge is several orders of magnitude smaller than the integral time scale of the turbulence. This would need however to be re-examined for realistic airfoils where the leading edge has a finite radius of curvature, for instance using experimental data from Paterson \& Amiet [35], Devenport et al. [36] or Gruber \& Joseph [37, 38]. Note that although all the validation examples presented here are against analytical solutions, comparisons with experimental data have been presented in [20], but they were not sufficiently conclusive as far as the effect of the integral time scale is concerned, mainly because the numerical simulations were using a flat plate.

Indeed, applying the proposed method to a real airfoil would be important to include such geometry effects and more realistic mean flows. This would not involve any significant changes for the linearized Euler solver, apart from using body-fitted curvilinear grids. For the RPM however, one will have to track the random particles through a non-uniform mean flow which could be provided by a RANS simulation for instance.

\section{Acknowledgments}

This work was supported by the Engineering and Physical Science Research Council, UK (grant EP/F005741/1) and by Rolls-Royce plc through the Rolls-Royce University Technology Centre in Gas Turbine Noise at the Institute of Sound and Vibration Research.

\section{References}

\section{References}

[1] Kraichnan, R. H., "Diffusion by a Random Velocity Field," The Physics of Fluids, Vol. 13, No. 1, 1970, pp. 22-31.

[2] Bailly, C., Lafon, P., and Candel, S. M., "Stochastic Approach to Compute Subsonic Noise Using Linearized Euler's Equations," 20 ${ }^{\text {th }}$ AIAA Aeroacoustics Conference, Greater Seatle, Washington, May 1999, AIAA paper 1999-1872.

[3] Bechara, W., Bailly, C., and Lafon, P., "Stochastic Approach to Noise Modelling for Free Turbulent Flows," AIAA Journal, Vol. 32, No. 3, March 1994, pp. $455-463$.

[4] Billson, M., Eriksson, L.-E., and Davidson, L., "Jet Noise Prediction Using Stochastic Turbulence Modelling," 24 ${ }^{\text {th }}$ AIAA Aeroacoustics Conference, Hilton Head, South California, May 2003, AIAA paper 2003-3282.

[5] Omais, M. and Caruelle, B., "Jet Noise Prediction Using RANS CFD Input," $29^{\text {th }}$ AIAA Aeroacoustics Conference, Vancouver, Canada, May 2008, AIAA paper 2008-2938.

[6] Casper, J. and Farassat, F., “A Time Domain Formulation for Broadband Noise Predictions," Aeroacoustics, Vol. 1, No. 3, 2002 , pp. $207-240$.

[7] Fang, J. and Atassi, H. M., "Numerical Solutions for Unsteady Subsonic Vortical Flows Around Loaded Cascades," Journal of Turbomachinery, Vol. 115, October 1993, pp. 810-816.

[8] Atassi, H. M., Ali, A. A., Atassi, O. V., and Vinogradov, I. V., "Scattering of Incident Disturbances by an Annular Cascade in a Swirling Flow," J. Fluid Mech., Vol. 499, 2004, pp. 111-138.

[9] Atassi, H. M. and Logue, M. M., "Effect of Turbulence Structure on Broadband Fan Noise," $29^{\text {th }}$ AIAA Aeroacoustics Conference, Vancouver, Canada, May 2008, AIAA paper 2008-2842.

[10] Careta, A., Sagues, F., and Sancho, J., "Stochastic Generation of Homogeneous Isotropic Turbulence with Well-Defined Spectra," Physical Review E, Vol. 48, No. 3, 1993, pp. 2279-2287. 
[11] Ewert, R. and Emunds, R., "CAA Slat Noise Studies Applying Stochastic Sound Sources Based On Solenoidal Digital Filters," $26^{\text {th }}$ AIAA Aeroacoustics Conference, Monterey, California, May 2005, AIAA paper 2005-2862.

[12] Klein, M., Sadiki, A., and Janicka, J., "A Digital Filter Based Generation of Inflow Data for Spatially Developing Direct Numerical or Large Eddy Simulations," Journal of computational Physics, Vol. 186, 2003, pp. 152-665.

[13] Ewert, R., Dierke, J., Siebert, J., Neifeld, A., Appel, C., Siefert, M., and Kornow, O., "CAA Broadband Noise Prediction for Aeroacoustic Design," Journal of Sound and Vibration, Vol. 330, No. 17, 2011, pp. 4139-4160.

[14] Ewert, R., Kornow, O., Tester, B. J., Powles, C. J., Delfs, J. W., and Rose, M., "Spectral Broadening of Jet Engine Turbine Tones," $29^{t h}$ AIAA Aeroacoustics Conference, Vancouver, Canada, May 2008, AIAA paper 2008-2940.

[15] Ewert, R., "Broadband Slat Noise Prediction Based on CAA and Stochastic Sound Sources from a Random-Particle Mesh (RPM) Method," Computers and Fluids, Vol. 37, 2008, pp. 369-387.

[16] Siefert, M. and Ewert, R., "Sweeping Sound Generation in Jets Realized with a Random Particle-Mesh Method," $30^{\text {th }}$ AIAA Aeroacoustics Conference, Miami, USA, May 2009, AIAA paper 2009-3369.

[17] Ewert, R., "Slat Noise Trend Predictions Using CAA with Stochastic Sound Sources from a Random Particle Mesh Method (RPM)," 27 AIAA Aeroacoustics Conference, Cambridge, Massachusetts, May 2006, AIAA paper 2006-2667.

[18] Amiet, R. K., "Acoustic Radiation from an Airfoil in Turbulent Stream," Journal of Sound and Vibration Research, Vol. 41, No. 4, 1975, pp. 407-420.

[19] Dieste, M. and Gabard, G., "Broadband fan interaction noise using synthetic inhomogeneous non-stationary turbulence," $32^{\text {nd }}$ AIAA Aeroacoustics Conference, Portland, OR, USA, June 2011, AIAA paper 2011-2708.

[20] Dieste, M., Random-particle methods for broadband fan interaction noise, Ph.D. thesis, Institute of Sound and Vibration research, University of Southampton, UK, 2011.

[21] Ewert, R., "RPM - The Fast Random Particle-Mesh Method to Realize Unsteady Turbulent Sound sources and Velocity Fields for CAA Applications," $28^{\text {th }}$ AIAA Aeroacoustics Conference, Rome, Italy, May 2007, AIAA paper 2007-3506.

[22] Abramowitz, M. and Stegun, I., Handbook of mathematical functions, National Bureau of Standards, 1964.

[23] Siefert, M., Ewert, R., and Unruh, O. H. O., "A synthetic wall pressure model for the efficient simulation of boundary layer induced cabin noise," 16th AIAA/CEAS Aeroacoustics Conference, Stockholm, Sweden, 2010, AIAA paper 2010-3760.

[24] Noble, B., Methods based on the Wiener-Hopf technique, Pergamon Press, London, 1958.

[25] Cottet, G.-H. and Koumoutsakos, P., Vortex methods: theory and practice, Cambridge University Press, 2000.

[26] Kloeden, P. and Platen, E., Numerical Solution of Stochastic Differential Equations, Springer, 1992.

[27] Pope, S. B., Turbulent Flows, Cambridge University Press, 2000.

[28] Krasnoff, E. and Peskin, R., "The Langevin Model for Turbulent Diffusion," Geophysical Fluid Dynamics, Vol. 2, 1971, pp. 123-146.

[29] Tam, C. and Webb, J., "Dispersion-relation-preserving finite difference schemes for computational acoustics," Journal of Computational Physics, Vol. 107, 1993, pp. 262-281.

[30] Berland, J., Bogey, C., Marsden, O., and Bailly, C., "High-order, low dispersive and low dissipative explicit schemes for multiple-scale and boundary problems," Journal of Computational Physics, Vol. 224, 2007, pp. 637-662.

[31] Bogey, C. and Bailly, C., "A family of low dispersive and low dissipative explicit schemes for flow and noise computations," Journal of Computational Physics, Vol. 194, 2004, pp. 194-214.

[32] Karni, S., "Far-field filtering operators for suppression of reflections from artificial boundaries," SIAM Journal of Numerical Analysis, Vol. 33, No. 3, 1996, pp. 1014-1047.

[33] Kim, J. and Lee, D., "Generalized characteristics boundary conditions for computational aeroacoustics," AIAA Journal, Vol. 38, No. 11, 2000, pp. 2040-2049.

[34] Percival, D. and Walden, A., Spectral Analysis for Physical Applications: Multitaper and Conventional Univariate Techniques, Cambridge University Press, 1993.

[35] Paterson, R. W. and Amiet, R. K., "Noise and Surface Pressure Response of an Airfoil to Incident Turbulence," J. Aircraft, Vol. 14, No. 8, August 1977, pp. 729-736.

[36] Devenport, W. J., Staubs, J. K., and Glegg, S. A. L., "Sound radiation from real airfoils in turbulence," Journal of Sound and Vibration, Vol. 329, 2010, pp. 3470-3483.

[37] Gruber, M., Joseph, P., and Chong, T., "Experimental Investigation of Airfoil Self Noise and Turbulent Wake Reduction by the use of Trailing Edge Serrations," 16th AIAA/CEAS Aeroacoustics Conference, Stockholm, Sweden, 2010, AIAA paper 2010-3803.

[38] Gruber, M. and Joseph, P., "On the mechanisms of serrated airfoil trailing edge noise reduction," 17th AIAA/CEAS Aeroacoustics Conference, Portland, Oregon, USA, 2011, AIAA paper 2011-2781. 

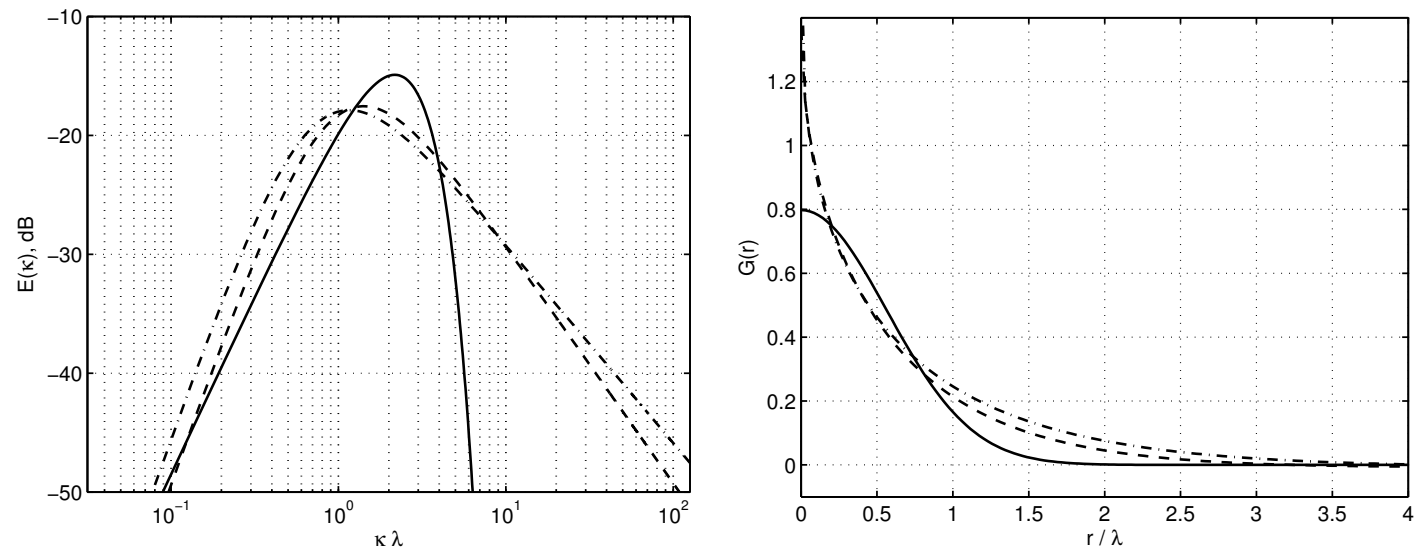

Figure 1: Left: Illustration of the three energy spectra considered in this work for the same values of $K$ and $\lambda$ : Gaussian spectrum (solid line), Liepmann spectrum (dashed line) and von Kármán spectrum (dot-dashed line). Right: Filters for the Gaussian spectrum (solid line), Liepmann spectrum (dashed line) and von Kármán spectrum (dot-dashed line).

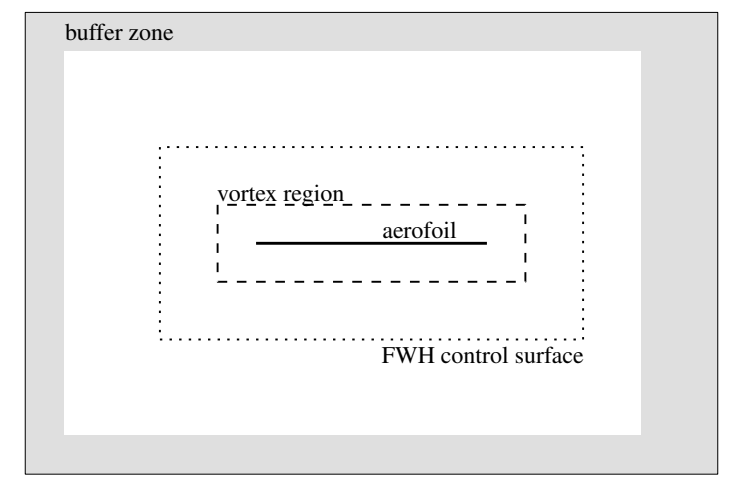

Figure 2: Diagram of the computational domain (the uniform mean flow is from left to right). 

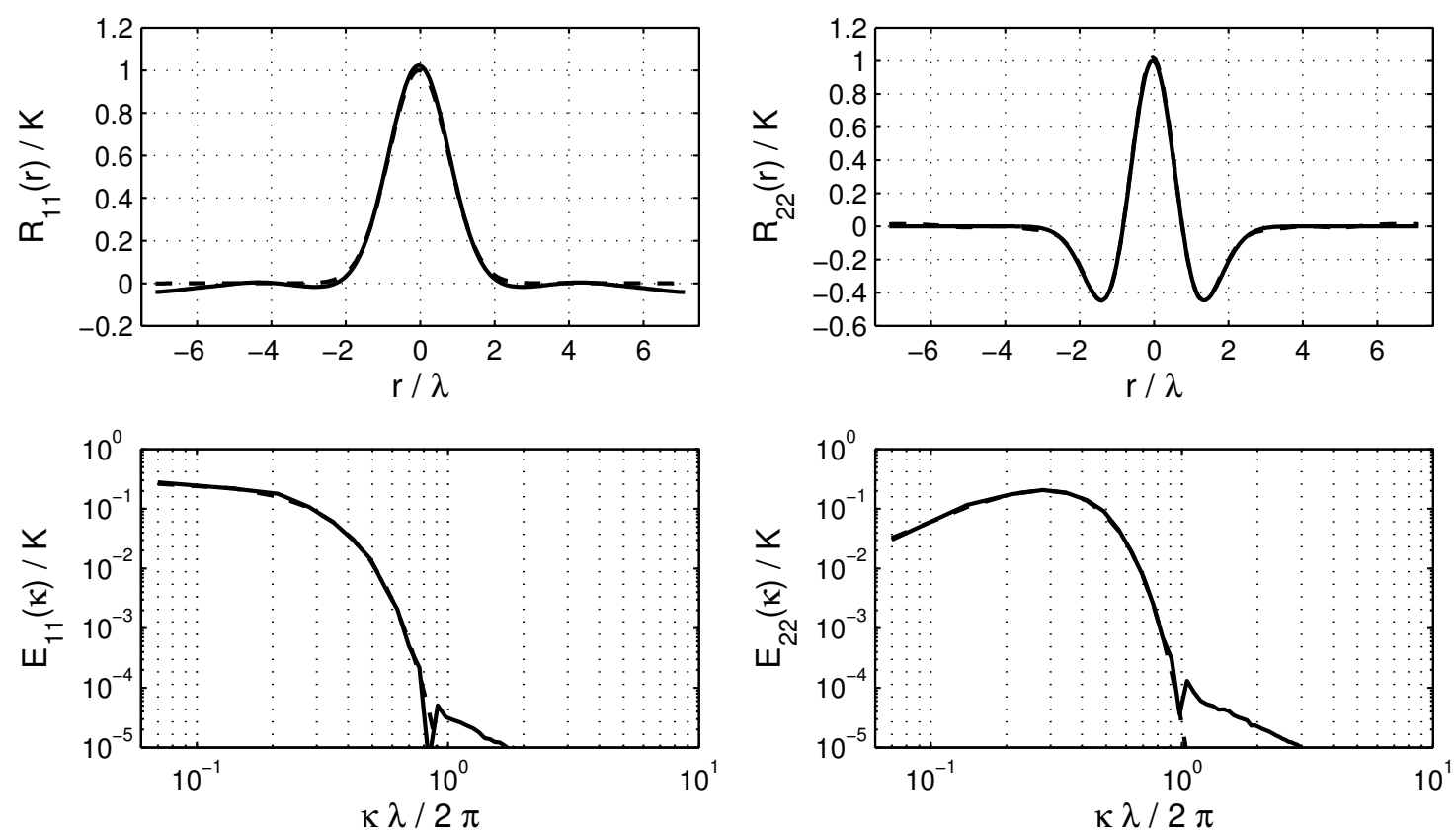

Figure 3: Analytical solution (dashed line) and numerical results (solid line) obtained with the Gaussian filter. Top: normalized correlations $R_{11}$ and $R_{22}$ computed at the center of the airfoil. Bottom: normalized one-dimensional energy spectra $E_{11}$ and $E_{22}$.
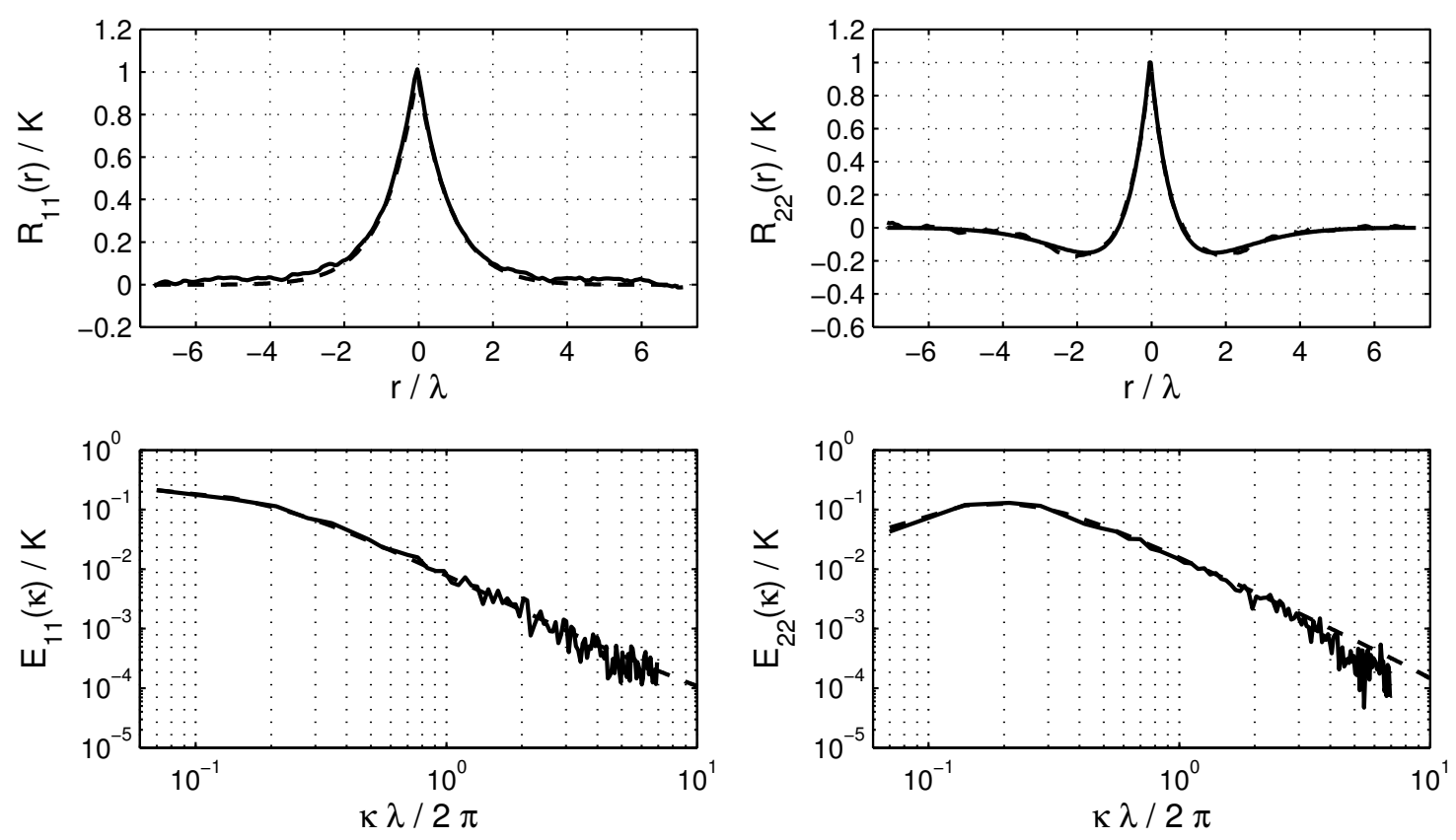

Figure 4: Analytical solution (dashed line) and numerical results (solid line) obtained with the Liepmann filter. Top: normalized correlations $R_{11}$ and $R_{22}$ computed at the center of the airfoil. Bottom: normalized one-dimensional energy spectra $E_{11}$ and $E_{22}$. 

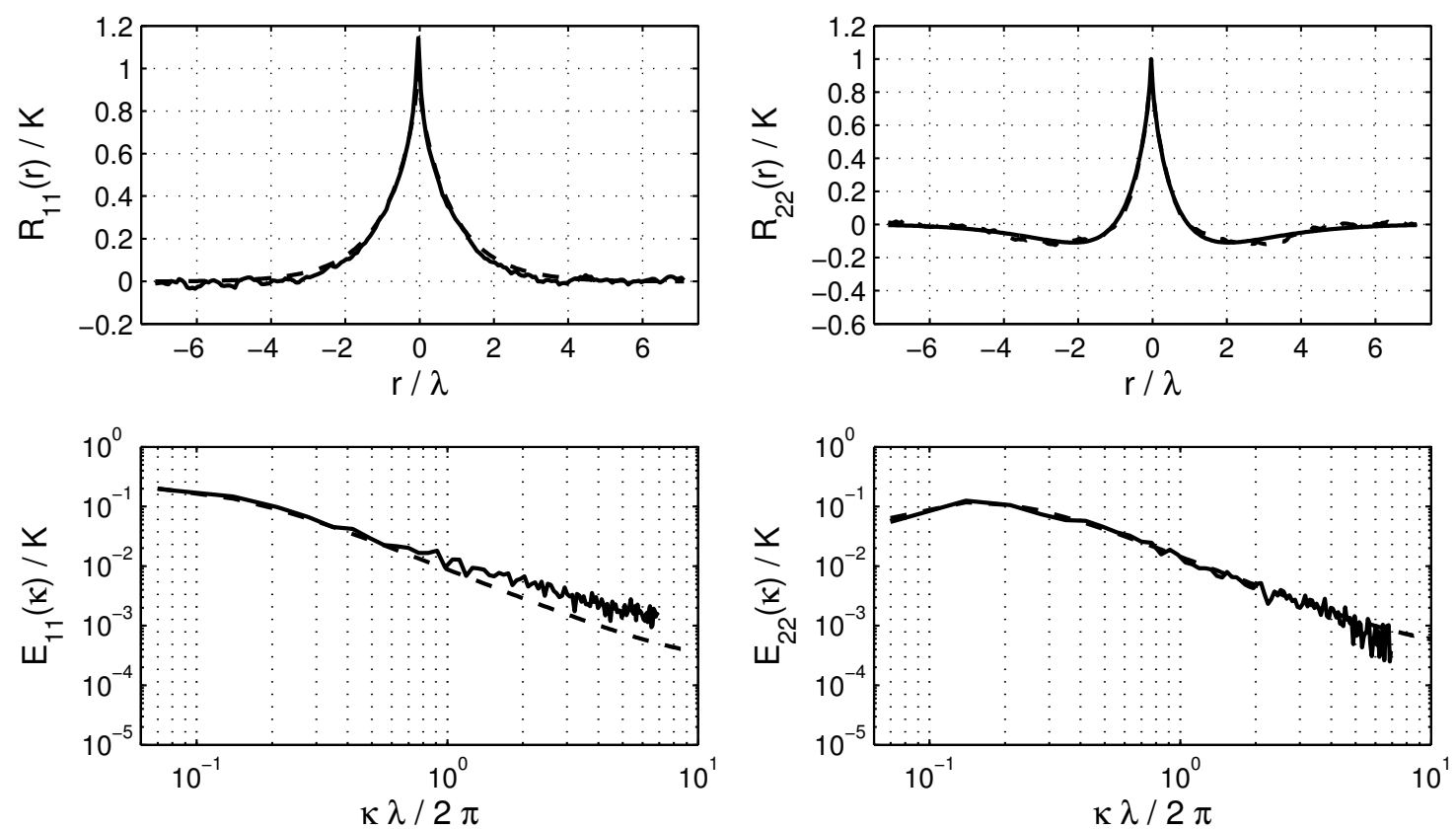

Figure 5: Analytical solution (dashed line) and numerical results (solid line) obtained with the von Kármán filter. Top: normalized correlations $R_{11}$ and $R_{22}$ computed at the center of the airfoil. Bottom: normalized one-dimensional energy spectra $E_{11}$ and $E_{22}$.

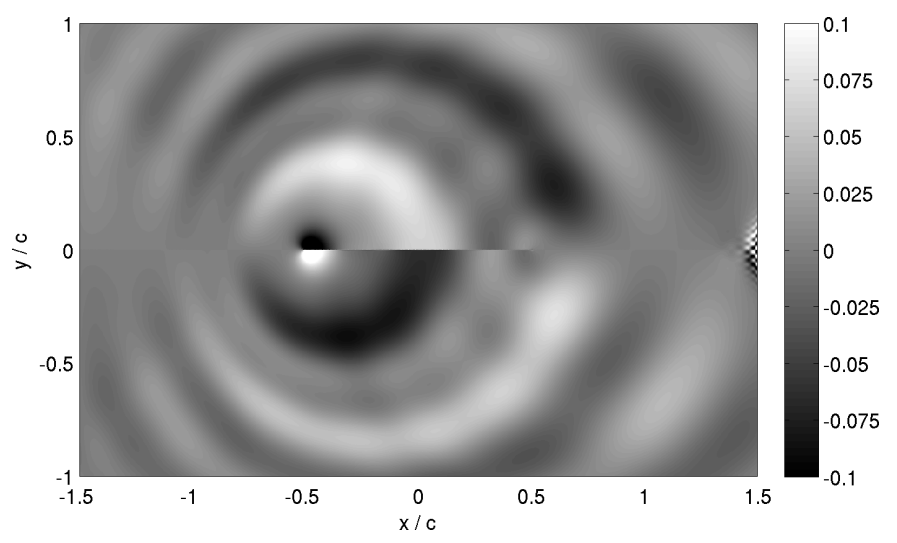

Figure 6: Snapshot of the acoustic pressure in the near field for the Gaussian spectrum. 

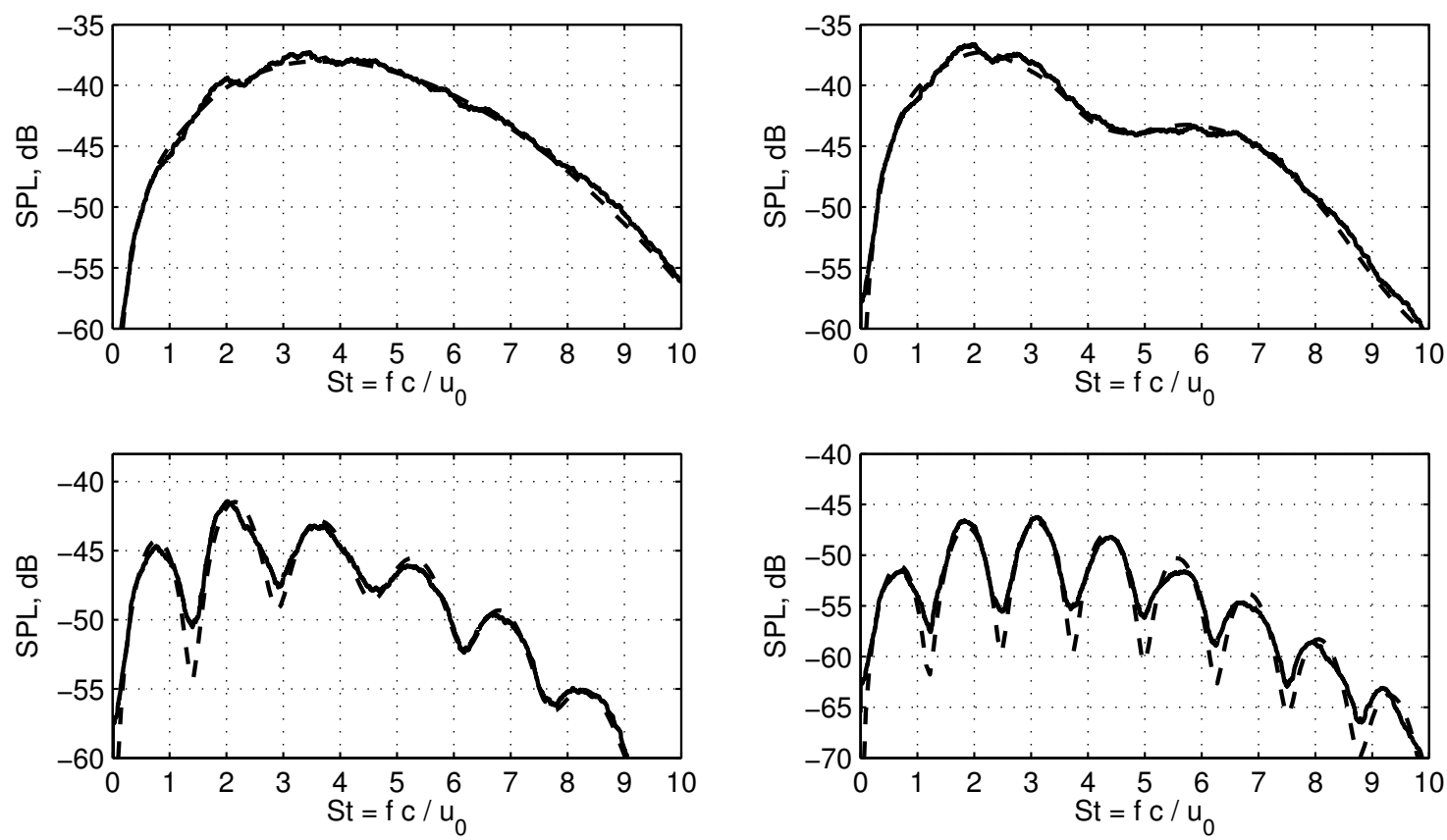

Figure 7: Theoretical (dashed line) and numerical (solid line) sound pressure levels in the far field obtained with the Gaussian spectrum. Observers located at $30^{\circ}$ (top left), $60^{\circ}$ (top right), $120^{\circ}$ (bottom left), and $150^{\circ}$ (bottom right).
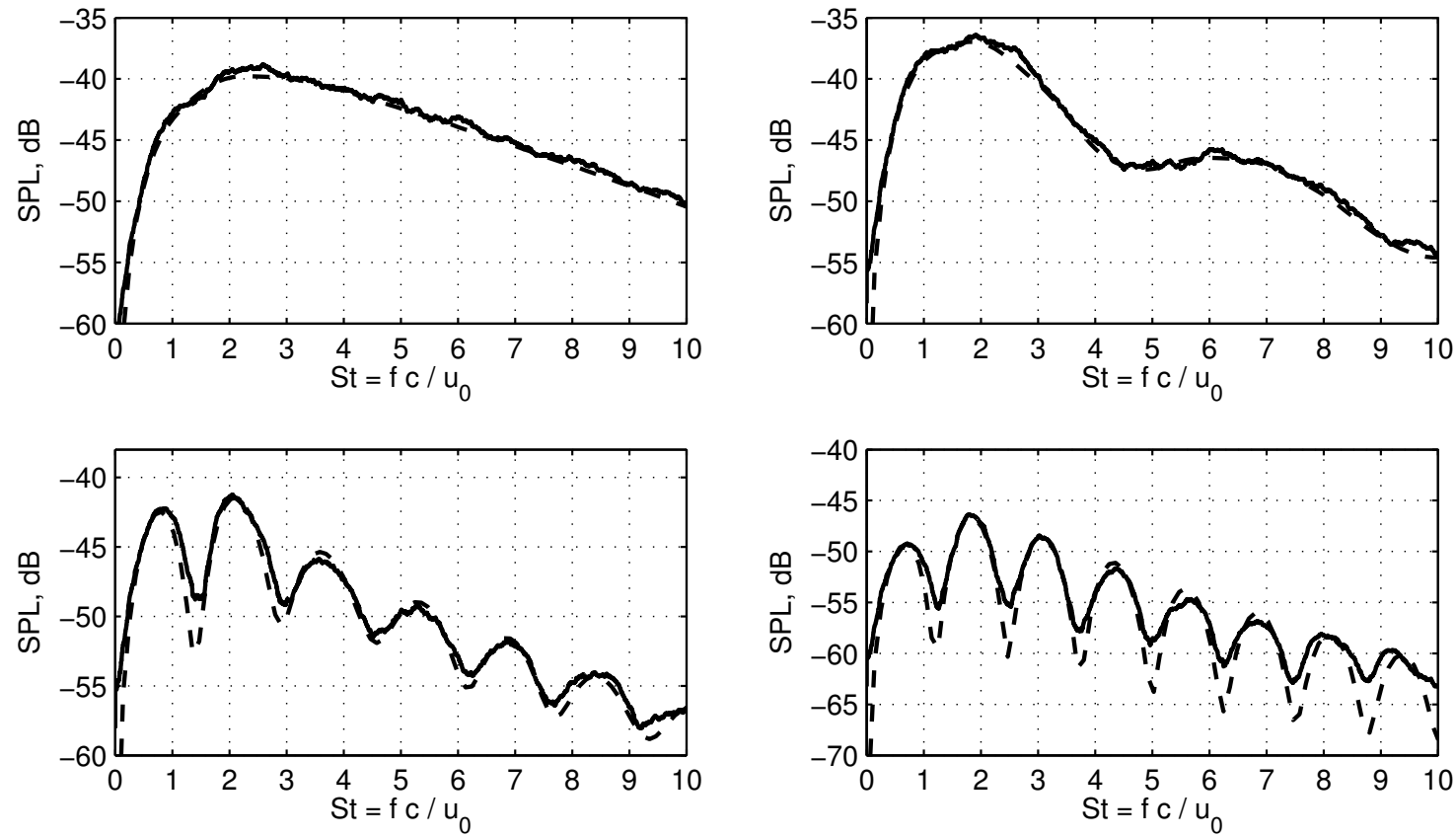

Figure 8: Theoretical (dashed line) and numerical (solid line) sound pressure levels in the far field obtained with the Liepmann spectrum. Observers located at $30^{\circ}$ (top left), $60^{\circ}$ (top right), $120^{\circ}$ (bottom left), and $150^{\circ}$ (bottom right). 

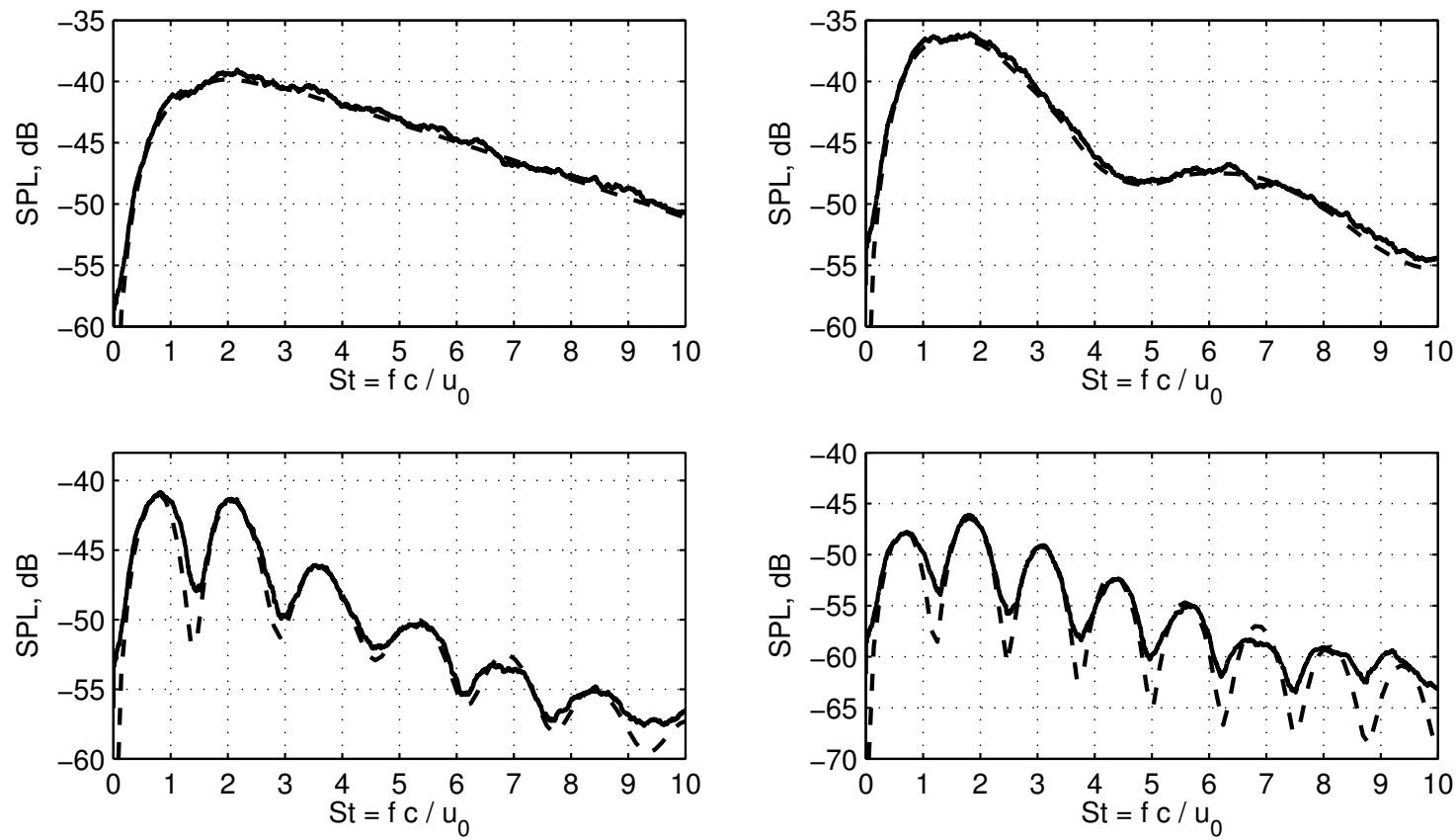

Figure 9: Theoretical (dashed line) and numerical (solid line) sound pressure levels in the far field obtained with the von Kármán spectrum. Observers located at $30^{\circ}$ (top left), $60^{\circ}$ (top right), $120^{\circ}$ (bottom left), and $150^{\circ}$ (bottom right).
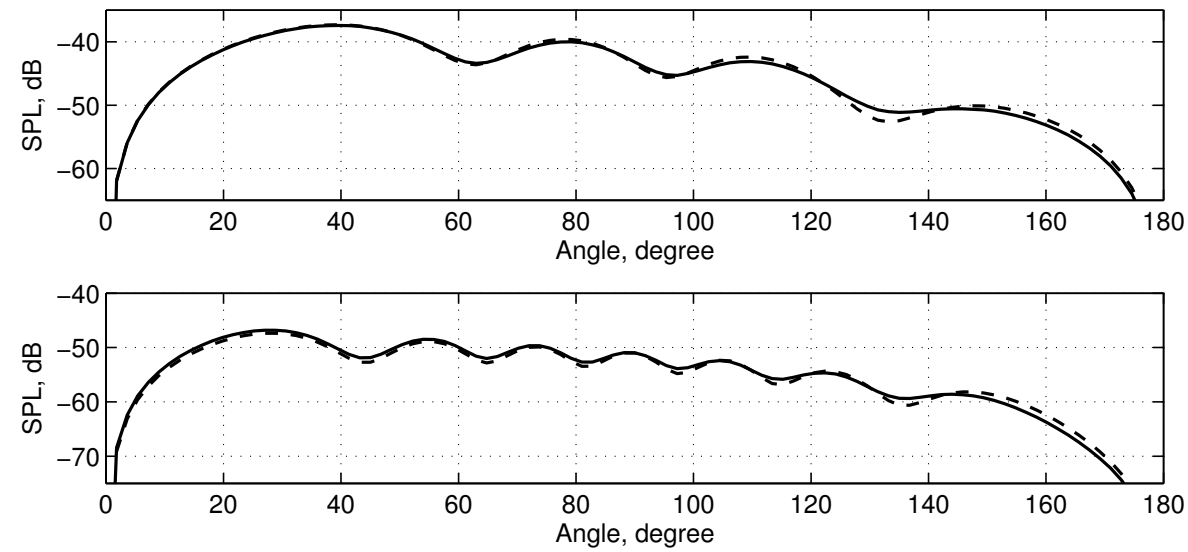

Figure 10: Theoretical (dashed line) and numerical (solid line) far-field directivity obtained with the Gaussian filter for $S t=2.03$ (top) and $S t=4.06$ (bottom). 

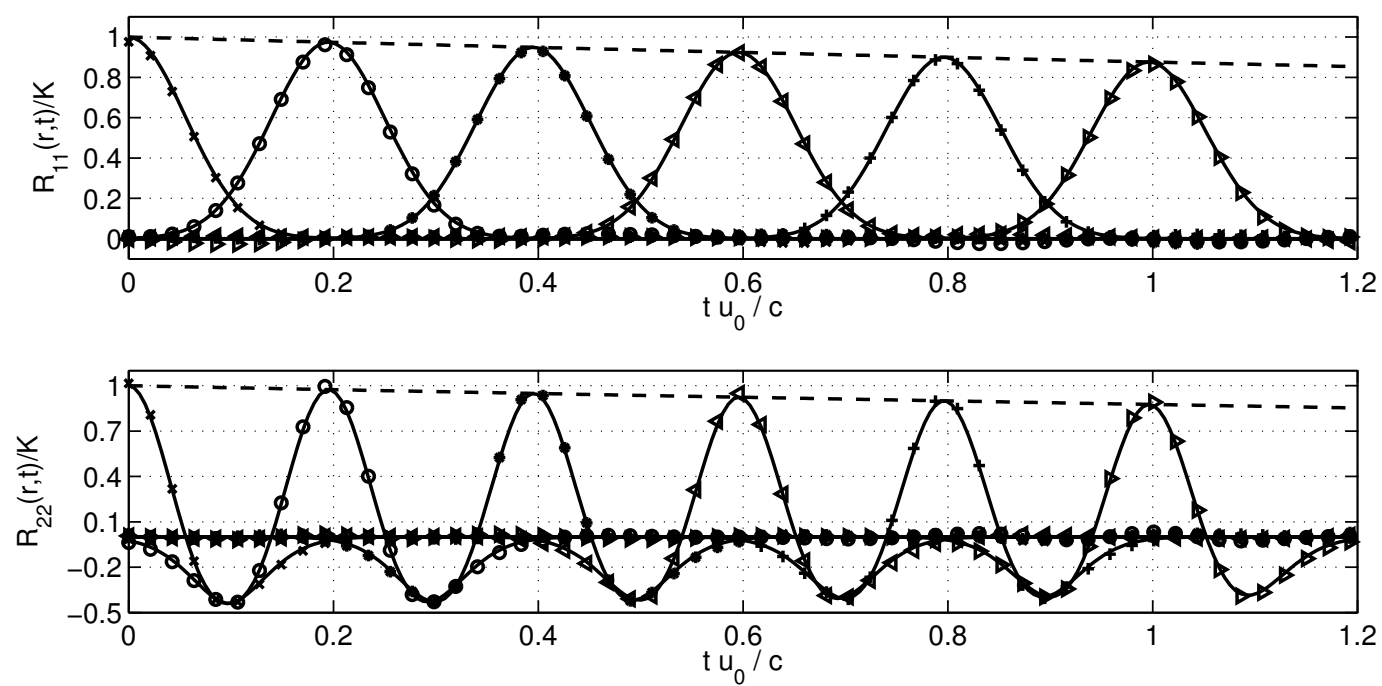

Figure 11: Two-point space-time correlations $R_{11}$ (top) and $R_{22}$ (bottom) for spatial separations $r / b=0(\times), r / b=0.2(\circ), r / c=0.4(*), r / c=0.6$ $(\triangleleft), r / c=0.8(+)$, and $r / c=1(\triangleright)$. Solid lines represent the analytical results and symbols numerical results. The dashed line $\operatorname{shows}$ the $\exp \left(-t / T_{L}\right)$ temporal decorrelation.
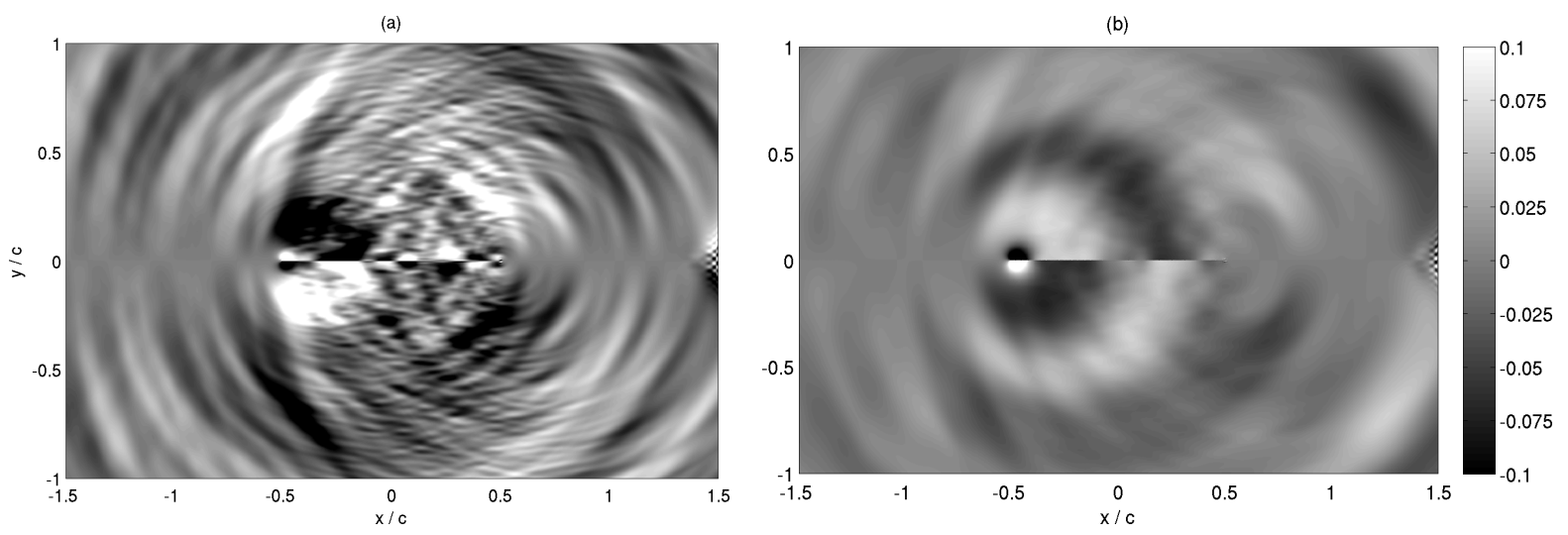

Figure 12: Snapshot of the acoustic pressure in the near field with the Gaussian spectrum, using the standard Langevin equation (a), or the secondorder Langevin equation (b). 
(a)

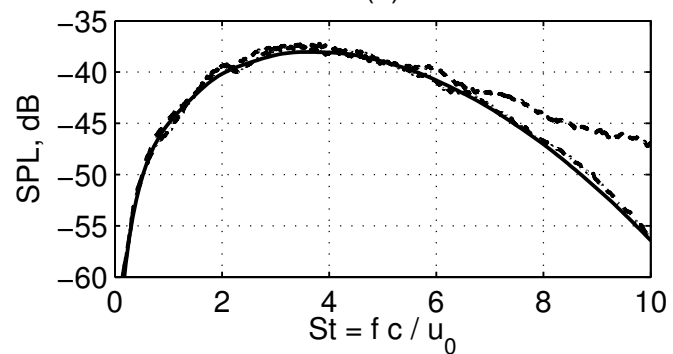

(b)

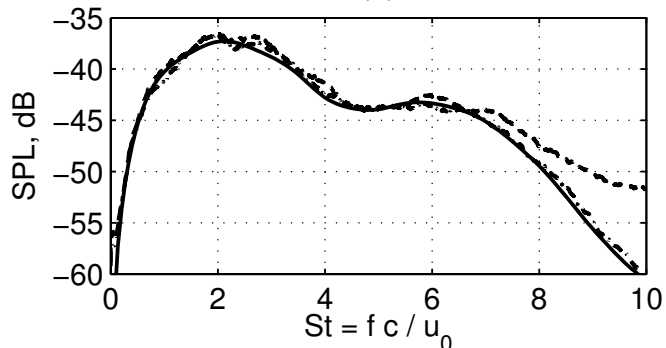

(c)

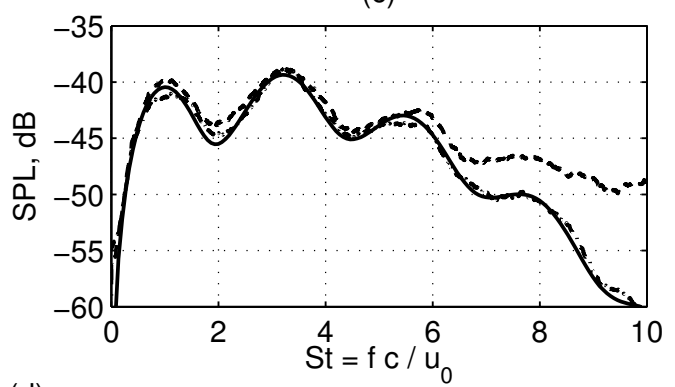

(d)

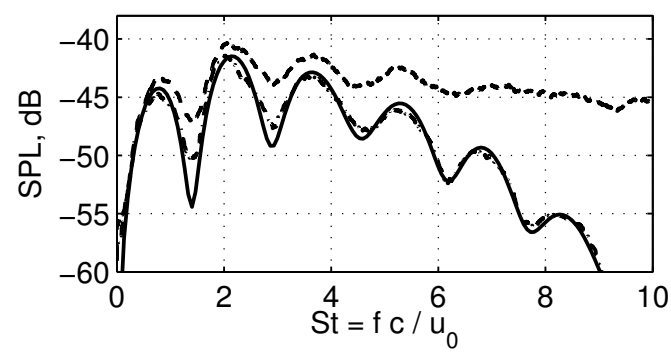

(e)

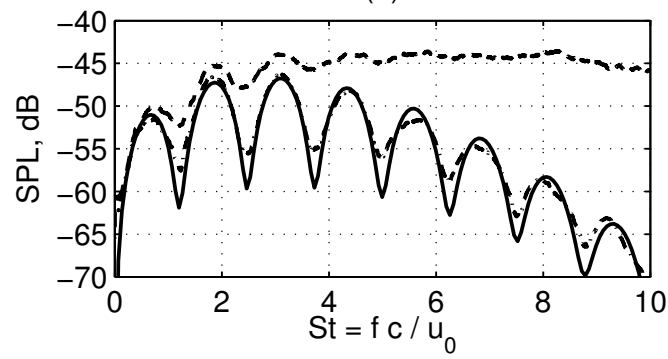

Figure 13: Normalized sound pressure levels in the far field at $30^{\circ}$ (a), $60^{\circ}$ (b), $90^{\circ}$ (c), $120^{\circ}$ (d), and $150^{\circ}$ (e). Solid line: analytical model for evolving turbulence. Dot-dashed line: numerical results for frozen turbulence. Dashed line: numerical results for evolving turbulence.
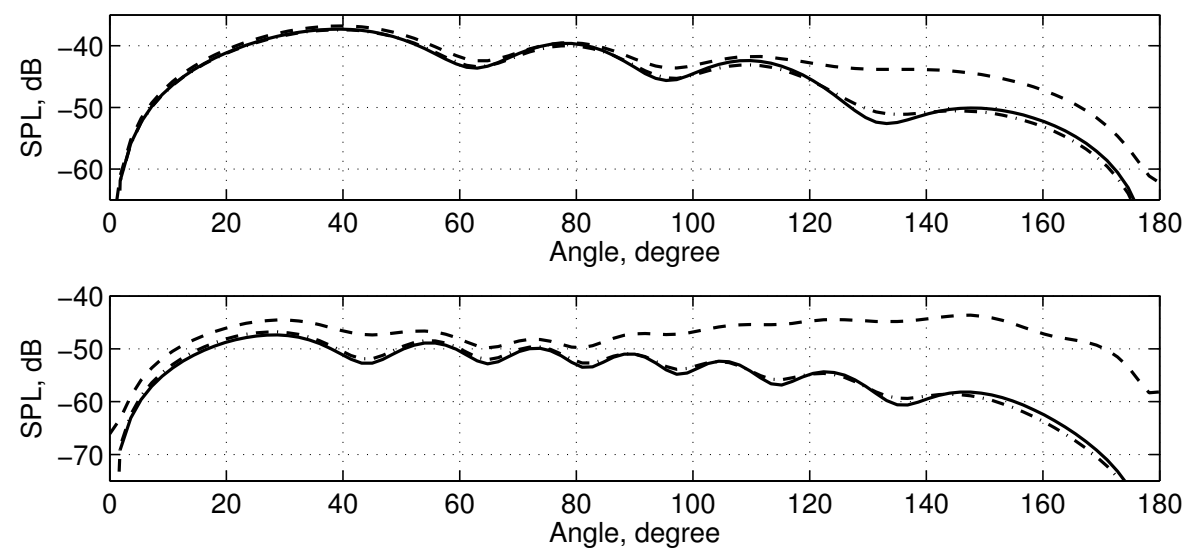

Figure 14: Sound pressure directivity in the far field at $S t=2.03$ (top) and $S t=4.06$ (bottom). Solid line: analytical model for evolving turbulence. Dot-dashed line: numerical results for frozen turbulence. Dashed line: numerical results for evolving turbulence. 
(a)

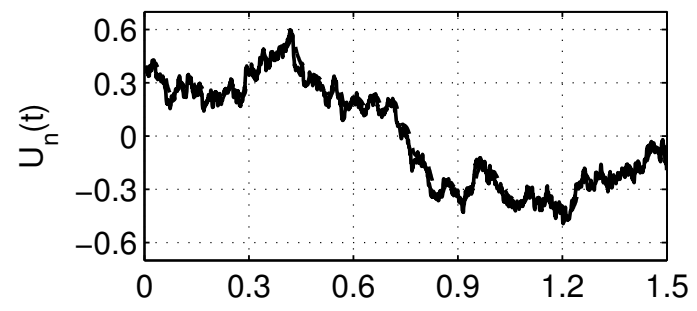

(c)

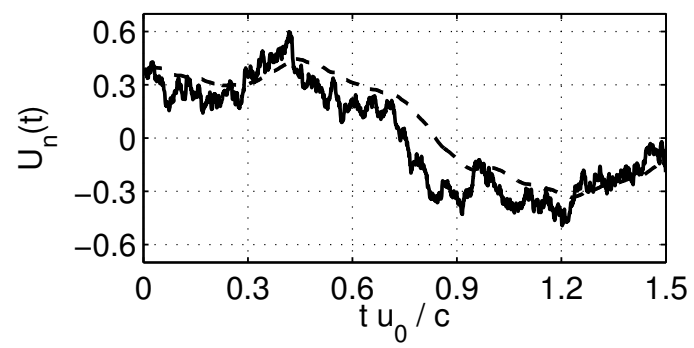

(b)

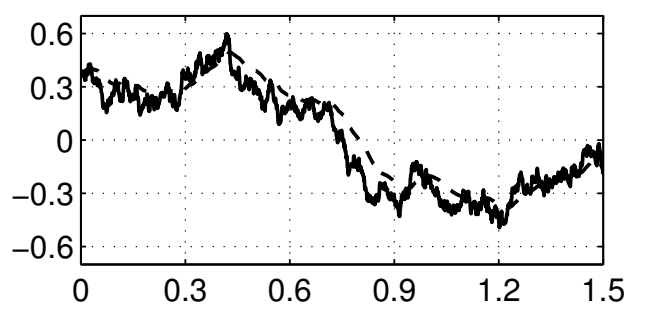

(d)

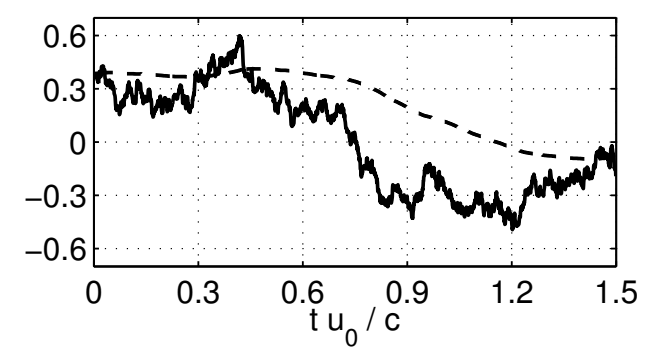

Figure 15: Example of time evolution of the strength of a vortex particle modelled by the Langevin equation (17) versus the strength modelled by the second-order Langevin model (25). From top to bottom and left to right, $T_{L} / \tau_{d} \approx 700, T_{L} / \tau_{d} \approx 440, T_{L} / \tau_{d} \approx 70$, and $T_{L} / \tau_{d} \approx 20$. 
(a)

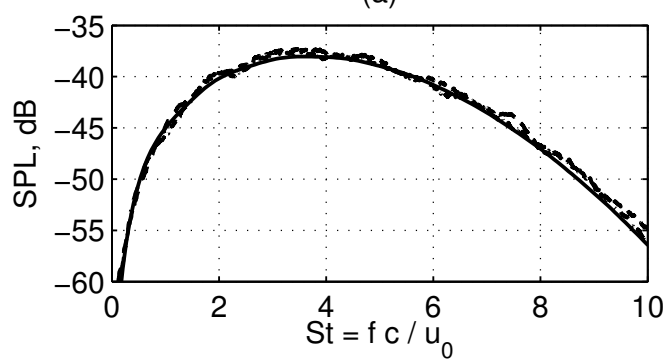

(b)

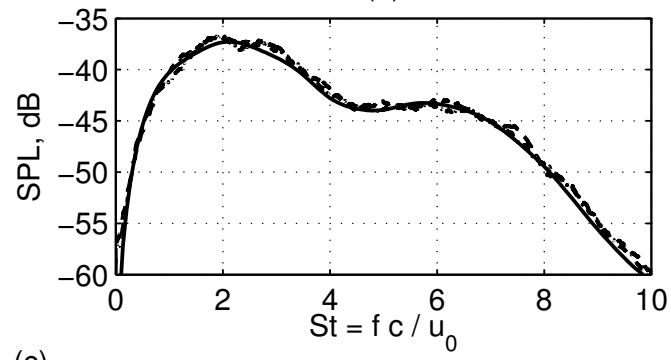

(c)

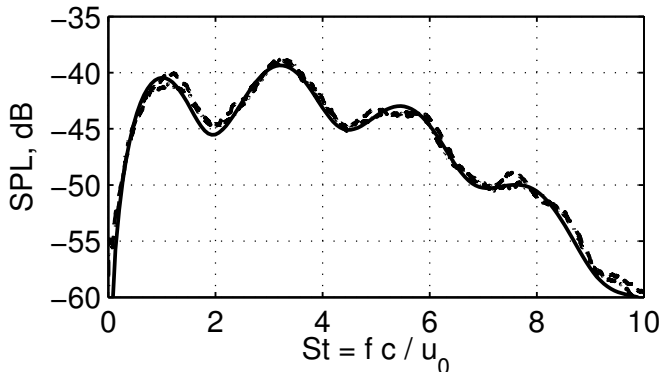

(d)

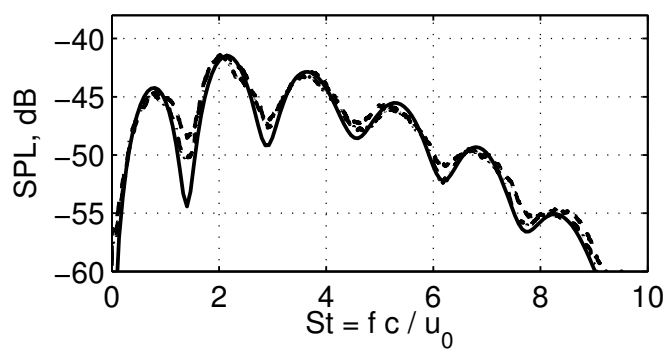

(e)

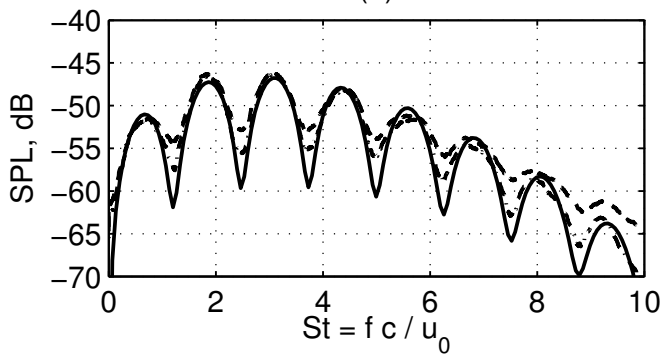

Figure 16: Normalized sound pressure levels in the far field at $30^{\circ}$ (a), $60^{\circ}$ (b), $90^{\circ}$ (c), $120^{\circ}$ (d), and $150^{\circ}$ (e). Solid line: analytical model for evolving turbulence. Dot-dashed line: numerical results for frozen turbulence. Dashed line: numerical results for evolving turbulence using the second-order Langevin equation.
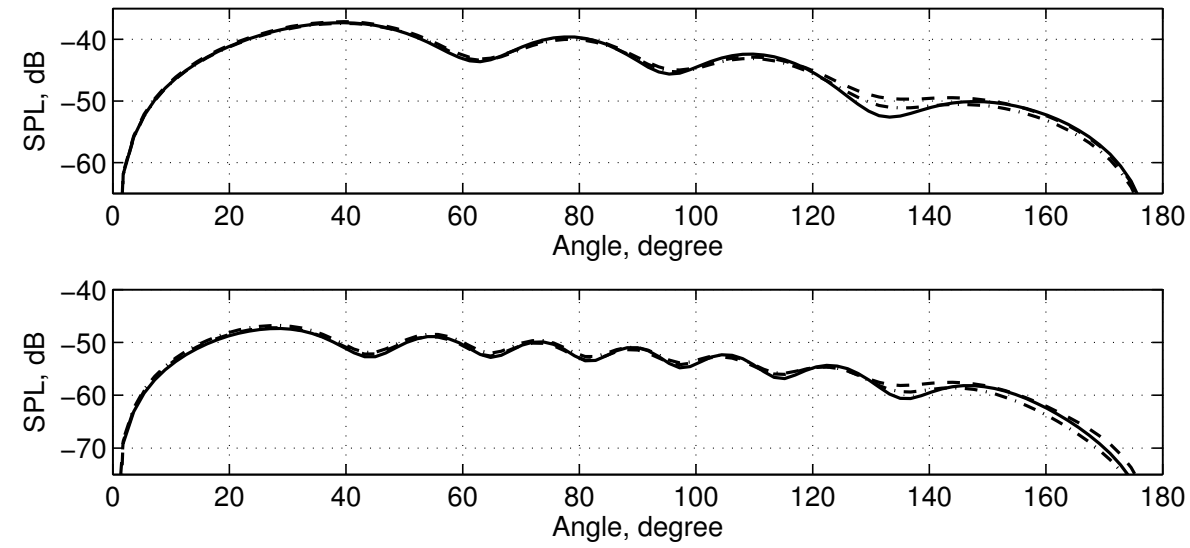

Figure 17: Sound pressure directivity in the far field at $S t=2.03$ (top) and $S t=4.06$ (bottom). Solid line: analytical model for evolving turbulence. Dot-dashed line: numerical results for frozen turbulence. Dashed line: numerical results for evolving turbulence using the second-order Langevin equation. 